\title{
A Non-Random Optimization Approach to a Disposal Mechanism Under Flexibility and Reliability Criteria
}

\author{
P.K. Tripathy ${ }^{1}$ and M. Pattnaik ${ }^{*}, 2$ \\ ${ }^{I}$ P.G. Department of Statistics, Utkal University, Bhubaneswar-751004, India \\ ${ }^{2}$ Department of Business Administration, Utkal University, India
}

\begin{abstract}
This paper focuses on an optimal disposal mechanism by extending the work of Tripathy et al. [11] to consider the system cost as fuzzy under flexibility and reliability criteria. It has been observed that by using a non-random optimization technique the derived disposal plan may result in no substantial difference in the system cost between the crisp and fuzzy model. Various numerical analyses demonstrate the effectiveness of proposed method. The results of the best order size obtained by the robust study are in general, very satisfactory. Finally through numerical examples sensitivity analysis show the influence of key model parameters. Based on consistency study, fuzzy strategy is better off than that of fixed cost strategy. Through sensitivity and robust analysis the results indicate that the performance of the proposed approach is superior to that of its crisp model.
\end{abstract}

AMS (2000) Subject classification No: 90B05.

Keywords: Fuzzy, reliability, flexibility.

\section{INTRODUCTION}

The EOQ model is a simple mathematical model to deal with inventory management issues in a production inventory system. It is considered to be one of the most popular inventory models used in the industry, see Silver [1], Silver et al. [2], Urgelleitti [3], Wagner [4], and Whitin [5]. However, these models are developed under many restrictive assumptions. There are inexhaustible number of studies that focus on imperfect production processes in inventory management, see Cheng [6, 7], Hadley et al. [8], Porteus [9], Rosenblatt et al. [10], Tapiero et al. [11] and Tripathy et al. [12] in which the items produced are of perfect quality but in reality product quality is never perfect with the production process and quality assurance established to monitor the product quality. Many studies approach this problem in a fuzzy framework, see Lee et al. [13], Vujosevic et al. [14] and Zimmermann [15]. Although there is some literature focussing attention on analytical approaches, Tripathy et al. [16] however, concentrated on using fuzzy optimization techniques for making in a disposal mechanism under flexibility and reliability criteria. Through very representative data our analyses show how such an approach can significantly improve fuzzy optimization in disposal mechanism. It will also show how a decision maker can dynamically access the various strategies as more information will become available during the optimization process. It is believed that the approach will be appealing because of its case of implementation. In real life situation uncertainty arises in decision making problem due to either

*Address correspondence to this author at the Department of Business Administration, Utkal University, India; Tel: 08895436436, 09937484316; E-mail: monalisha_1977@yahoo.com lack of knowledge or inherent vagueness. In this paper economic order quantity in the fuzzy sense is tied with process flexibility and reliability considerations. The approach presented here is different from Tripathy et al. [16] in which the total cost function is expressed as:

$$
\begin{aligned}
Z(r, \lambda, q)= & \frac{S r}{\lambda q}+\operatorname{ar}(1-r)^{c} \lambda^{-(b+1)}+\frac{H q}{2 r} \\
& =F_{1}+F_{2} S+F_{3} H
\end{aligned}
$$

where, $F_{1}=\operatorname{ar}(1-r)^{c} \lambda^{-(b+1)}, F_{2}=\frac{r}{\lambda q} \& F_{3}=\frac{q}{2 r}$

The EOQ which minimizes $Z(r, \lambda, q)$ in (1) is

$q^{*}=\sqrt{\frac{2 S}{\lambda H}} \times r$

where, $Z(r, \lambda, q)$ is the total relevant cost per unit time.

The method is then relaxed and two important costs affecting the output of inventory model, namely setup cost and inventory holding cost are assumed to be fuzzy. The motivation for the study lies in the author's observation that the setup and inventory holding cost are not precisely known in practice but subjectively estimated. This puts an observational bias incorporating errors to the total cost. The computational error can be greatly reduced by the procedure of defuzzifying the fuzzy variables to compute the total cost. There are two important aspects of working with this paper, the first one being related to the development of theoretical basis for the fuzzy procedure using the work of Mahata et al. [17] and the other one being the numerical evaluation of this fuzzy model as applied to crisp model of Tripathy et al. [16] exposing its relative efficiency. 
Table 1. Major Characteristics of Inventory Models on Selected Researches

\begin{tabular}{|c|c|c|c|c|c|c|}
\hline $\begin{array}{l}\text { Author(s) and } \\
\text { Published Year }\end{array}$ & $\begin{array}{c}\text { Structure } \\
\text { of the Model }\end{array}$ & Deterioration & $\begin{array}{l}\text { Production } \\
\text { Cost }\end{array}$ & $\begin{array}{c}\text { Simulated } \\
\text { Sample Studies }\end{array}$ & Demand Linked to Quality & Robust Analysis \\
\hline Cheng (1991) & Crisp & No & $\begin{array}{l}\text { Directly related to } \\
\text { process reliability and } \\
\text { demand is constant. }\end{array}$ & No & No & No \\
\hline Vujosevic et al. (1996) & Fuzzy & No & No & No & No & No \\
\hline Lee and Yao (1998) & Fuzzy & No & No & No & No & No \\
\hline Tripathy et al. (2003) & Crisp & No & $\begin{array}{l}\text { Directly related to } \\
\text { process reliability } \\
\text { and inversely related } \\
\text { to demand rate. }\end{array}$ & No & Yes & No \\
\hline $\begin{array}{c}\text { Mahata and } \\
\text { Goswami (2006) }\end{array}$ & Fuzzy & Yes & No & No & No & No \\
\hline Tripathy et al. (2009) & Crisp & No & $\begin{array}{l}\text { Inversely related } \\
\text { to both process } \\
\text { reliability and to } \\
\text { demand rate }\end{array}$ & No & Yes & No \\
\hline Present Paper (2009) & Fuzzy & No & $\begin{array}{l}\text { Inversely related } \\
\text { to both process } \\
\text { reliability and to } \\
\text { demand rate }\end{array}$ & Yes & Yes & Yes \\
\hline
\end{tabular}

In this paper a generalized mathematical model is developed in which the shortcomings of all the previous models are taken care of. The primary difference of this paper as compared to previous studies is that, we introduce a generalized inventory model by relaxing the traditional inventory model in different ways which are described in Table 1. We then establish several theoretical results to characterize the optimal solution under various situations and prescribe five numerical examples to illustrate the given model. The adoption of this method ultimately depends on its cost savings as well as sensitivity to values of various cost parameters.

\section{ASSUMPTIONS AND NOTATIONS}

Here in the model Tripathy et al. [16], demand for the product exceeds supply, all items are subjected to inspection and all defective items are discarded. The unit cost of production is inversely related to both process reliability and demand according to the general power function. $P(r, \lambda)=a(1-r)^{c} \lambda^{-b}$ where, $a, b$ and $c$ are nonnegative real numbers so as to be chosen to provide the best fit of the estimated cost function. $S$ and $H$ be the setup cost per production run and inventory carrying cost per unit time respectively. $q$ and $\lambda$ indicate production quantity per production run and demand respectively. $r$ and $p(r, \lambda)$ be the production process reliability and unit cost of production respectively. Similarly $Z(r, \lambda, q)$ and $M_{\tilde{T C}}(r, \lambda, q)$ be the total cost of setup, production and inventory holding per unit time in crisp and fuzzy strategy respectively.

\section{DESCRIPTION OF THE PROPOSED MODEL}

The proposed fuzzy model is the simplest and the most efficient, but the uncertainty itself is almost neglected. Since it calculates the fuzzy cost values and then determines the minimum of their defuzzifyied values. It requires less computational efforts for comparison of fuzzy numbers. The triangular fuzzy models are simple and there are no computational problems at all. However it is not easy to say which of the described models is the best or most appropriate. There is a general recommendation that it is always better to keep uncertainty in the model as long as possible. From that point of view the triangular fuzzy method is the best one. In any case one may opine that an analysis like the one presented in this paper provides the decision maker with a differ insight into the problem.

We replace the set up cost and inventory holding cost by fuzzy numbers $\tilde{S}$ and $\tilde{H}$ respectively. By expressing $\tilde{S}$ and $\tilde{H}$ as the normal triangular fuzzy numbers $\left(S_{1}, S_{0}, S_{2}\right)$ and $\left(H_{1}, H_{0}, H_{2}\right)$, where, $S_{1}=S-\Delta_{1}, \quad S_{0}=S, \quad S_{2}=S+\Delta_{2}, \quad H_{1}=H-\Delta_{3}$, $H_{0}=H, H_{2}=H+\Delta_{4}$ such that $0<\Delta_{1}<S, 0<\Delta_{2}, 0<\Delta_{3}<H, 0<\Delta_{4}$. $\Delta_{l}, \Delta_{2},, \Delta_{3}$ and $\Delta_{4}$ are determined by the decision maker based on the uncertainty of the problem.

The membership function of fuzzy set-up cost and fuzzy inventory holding cost are considered as:

$$
\mu_{\tilde{S}}(S)=\left\{\begin{array}{ccc}
\frac{S-S_{1}}{S_{0}-S_{1}} & ; & S_{1} \leq S \leq S_{0} \\
\frac{S_{2}-S}{S_{2}-S_{0}} & ; & S_{0} \leq S \leq S_{2} \\
0 & ; & \text { otherwise }
\end{array}\right.
$$




$$
\mu_{\tilde{H}}(H)=\left\{\begin{array}{ccc}
\frac{H-H_{1}}{H_{0}-H_{1}} & ; & H_{1} \leq H \leq H_{0} \\
\frac{H_{2}-H}{H_{2}-H_{0}} & ; & H_{0} \leq H \leq H_{2} \\
0 & ; & \text { other wise }
\end{array}\right.
$$

Then the centroid for $\tilde{S}$ and $\tilde{H}$ are given by

$M_{\tilde{S}}=\frac{S_{1}+S_{0}+S_{2}}{3}=S+\frac{\Delta_{2}-\Delta_{1}}{3}$ and

$M_{\tilde{H}}=\frac{H_{1}+H_{0}+H_{2}}{3}=H+\frac{\Delta_{4}-\Delta_{3}}{3}$ respectively.

For fixed values of $r, \lambda$ and $q$, let

$\mathrm{Z}(\mathrm{S}, \mathrm{H})=\mathrm{F}_{1}(\mathrm{r}, \lambda, \mathrm{q})+\mathrm{F}_{2}(\mathrm{r}, \lambda, \mathrm{q}) \mathrm{S}+\mathrm{F}_{3}(\mathrm{r}, \lambda, \mathrm{q}) \mathrm{H}=\mathrm{y}$.

Let $S=\frac{y-F_{1}-F_{2} H}{F_{2}}, \frac{\Delta_{2}-\Delta_{1}}{3}=\phi_{1}$ and $\frac{\Delta_{4}-\Delta_{3}}{3}=\phi_{2}$

By extension principle the membership of the fuzzy cost function is given by,

$$
\begin{aligned}
\mu_{\tilde{Z}(\tilde{S}, \tilde{H})}(y) & =\quad \operatorname{Sup}_{(S, H) \in Z^{-1}(y)}\left\{\mu_{\tilde{S}}(S) \wedge \mu_{\tilde{H}}(H)\right\} \\
& =\operatorname{Sup}_{H_{1} \leq H \leq H_{2}}\left\{\mu_{\tilde{S}}\left(\frac{y-F_{1}-F_{3} H}{F_{2}}\right) V \mu_{\tilde{H}}(H)\right\}
\end{aligned}
$$

Now,

$$
\mu_{\tilde{S}}\left(\frac{y-F_{1}-F_{3} H}{F_{2}}\right)=\left\{\begin{array}{ccc}
\frac{y-F_{1}-F_{2} S_{1}-F_{3} H}{F_{2}\left(S_{0}-S_{1}\right)} & ; u_{2} \leq H \leq u_{1} \\
\frac{F_{1}+F_{2} S_{2}+F_{3} H-y}{F_{2}\left(S_{2}-S_{0}\right)} & ; u_{3} \leq H \leq u_{2} \\
0 & ; \text { other wise }
\end{array}\right.
$$

where, $\quad . . \quad . . u_{1}=\frac{y-F_{1}-F_{2} S_{1}}{F_{3}}, \quad u_{2}=\frac{y-F_{1}-F_{2} S_{0}}{F_{3}} \quad$ and $u_{3}=\frac{y-F_{1}-F_{2} S_{2}}{F_{3}}$

When $u_{2} \leq H$ and $H \leq u_{1}$ then $y \leq F_{1}+F_{2} S_{0}+F_{3} H_{0}$ and $y \geq F_{1}+F_{2} S_{1}+F_{3} H_{1}$. It is clear that for every $y \in\left[F_{1}+F_{2} S_{1}+F_{3} H_{1}, F_{1}+F_{2} S_{0}+F_{3} H_{0}\right], \mu_{y}(y)=P Q$. From the equations 3 and 6 , the value of PQ may be found by solving the following equation:

$\frac{H-H_{1}}{H_{0}-H_{1}}=\frac{y-F_{1}-F_{2} S_{1}-F_{3} H}{F_{2}\left(S_{0}-S_{1}\right)}$

or $H=\frac{\left(y-F_{1}-F_{2} S_{1}\right)\left(H_{0}-H_{1}\right)+F_{2} H_{1}\left(S_{0}-S_{1}\right)}{F_{2}\left(S_{0}-S_{1}\right)+F_{3}\left(H_{0}-H_{1}\right)}$
Therefore,

$P Q=\frac{H-H_{1}}{H_{0}-H_{1}}=\frac{y-F_{1}-F_{2} S_{1}-F_{3} H_{1}}{F_{2}\left(S_{0}-S_{1}\right)+F_{3}\left(H_{0}-H_{1}\right)}=\mu_{1}(y)$,

When $u_{3} \leq H$ and $H \leq u_{2}$ then $y \leq F_{1}+F_{2} S_{2}+F_{3} H_{2}$ and $y \geq F_{1}+F_{2} S_{0}+F_{3} H_{0}$. It is evident that for every $y \in\left[F_{1}+F_{2} S_{0}+F_{3} H_{0}, F_{1}+F_{2} S_{2}+F_{3} H_{2}\right], \quad \mu_{\tilde{y}}(y)=P^{\prime} Q^{\prime}$. From the equations 3 and 6 , the value $\mathrm{P}^{\prime} \mathrm{Q}$ ' may be found by solving the following equation.

$\frac{H_{2}-H}{H_{2}-H_{0}}=\frac{F_{1}+F_{2} S_{2}+F_{3} H-y}{F_{2}\left(S_{2}-S_{0}\right)}$

or $H=\frac{F_{2} H_{2}\left(S_{2}-S_{0}\right)-\left(F_{1}+F_{2} S_{2}-y\right)\left(H_{2}-H_{0}\right)}{F_{2}\left(S_{2}-S_{0}\right)+F_{3}\left(H_{2}-H_{0}\right)}$

Therefore,

$P^{\prime} Q^{\prime}=\frac{H_{2}-H}{H_{2}-H_{0}}=\frac{F_{1}+F_{2} S_{2}+F_{3} H_{2}-y}{\left(S_{2}-S_{0}\right) F_{2}+\left(H_{2}-H_{0}\right) F_{3}}=\mu_{2}(y)$,

Thus the membership function for fuzzy total cost is given by

$\mu_{\tilde{Z} \tilde{\tilde{s}, \tilde{H}})}(y)=\left\{\begin{array}{cll}\mu_{1}(y) & ; \quad F_{1}+F_{2} S_{1}+F_{3} H_{1} \leq y \leq F_{1}+F_{2} S_{0}+F_{3} H_{0} \\ \mu_{2}(y) ; & F_{1}+F_{2} S_{0}+F_{3} H_{0} \leq y \leq F_{1}+F_{2} S_{2}+F_{3} H_{2} \\ 0 ; & \text { other wise }\end{array}\right.$

Now let $P_{1}=\int_{-\infty}^{\infty} \mu_{\tilde{Z} \tilde{\mathcal{S}}, \tilde{H})}(y) d y$ and $R_{1}=\int_{-\infty}^{\infty} y \mu_{\tilde{Z}(\tilde{S}, \tilde{H})}(y) d y$.

Hence the centroid for fuzzy total cost is given by

$M_{\tilde{T C}}(r, \lambda, q)=\frac{R_{1}}{P_{1}}=F_{1}(r, \lambda, q)+F_{2}(r, \lambda, q) S+F_{3}(r, \lambda, q) H$

$+\phi_{1} F_{2}(r, \lambda, q)+\phi_{2} F_{3}(r, \lambda, q)$

$M_{\tilde{T C}}(r, \lambda, q)=F_{1}+\left(S+\phi_{1}\right) F_{2}+\left(H+\phi_{2}\right) F_{3}$ subject to

$0 \leq r \leq 1$

Now for minimizing total average cost per unit time, the first partial derivative of $M_{\tilde{T C}}(r, \lambda, q)$ with respect to $r, \lambda$ and $q$ are set to zero to obtain the necessary optimality conditions:

$$
\begin{aligned}
& a \lambda^{-(b+1)}\left(\frac{(1-r)^{c}-r c(1-r)^{c-1}}{r^{2}}\right)+\frac{1}{\lambda q}\left(S+\phi_{1}\right)-\frac{q}{2 r^{2}}\left(H+\phi_{2}\right)=0 \\
& -a(b+1)(1-r)^{c} \lambda^{-(b+2)}-\frac{r}{\lambda^{2} q}\left(S+\phi_{1}\right)=0 \\
& -\frac{r}{\lambda q^{2}}\left(S+\phi_{1}\right)+\frac{1}{2 r}\left(H+\phi_{2}\right)=0
\end{aligned}
$$

from which $r=\frac{1}{c+1}$ 
$\lambda=\left[\frac{\left(S+\phi_{1}\right)\left(H+\phi_{2}\right)}{2 a^{2}(b+1)^{2}(1-r)^{2 c} r^{2}}\right]^{-\frac{1}{2 b+1}}$ and $q=\sqrt{\frac{2\left(S+\phi_{1}\right)}{\lambda\left(H+\phi_{2}\right)}} \times r$,

Hence,

$M_{\tilde{T C}}(r, \lambda, q)=\frac{a c^{c}}{(c+1)^{c+1}} \times L^{\frac{b+1}{2 b+1}}+\sqrt{2\left(S+\phi_{1}\right)\left(H+\phi_{2}\right)} \times L^{\frac{1}{2(2 b+1)}}$

where, $L=\frac{\left(S+\phi_{1}\right)\left(H+\phi_{2}\right)}{2 a^{2}(b+1)^{2} r^{2}(1-r)^{2 c}}$

\section{SPECIAL CASE}

After substituting the stationary points

$r^{*}=\frac{1}{c+1}$

$\lambda *=\left[\frac{\left(S+\phi_{1}\right)\left(H+\phi_{2}\right)(1+c)^{2(c+1)}}{2 a^{2}(b+1)^{2} \times c^{2 c}}\right]^{-\frac{1}{2 b+1}}$

and

$q^{*}=\frac{1}{(1+c)} \sqrt{\frac{2\left(S+\phi_{1}\right)}{\lambda^{*}\left(H+\phi_{2}\right)}}$

As shown in the Appendix-A, we derive the sufficient condition for stationary points $\left(r^{*}, \lambda^{*}, q^{*}\right)$ as

$\left|\begin{array}{ccc}f_{r^{* *}} & f_{r^{*} \lambda^{*}} & f_{r^{*} q^{*}} \\ f_{\lambda^{*} r^{*}} & f_{\lambda^{*} \lambda^{*}} & f_{\lambda^{*} q^{*}} \\ f_{q^{*} r^{*}} & f_{q^{*} \lambda^{*}} & f_{q^{*} q^{*}}\end{array}\right|>0 \Rightarrow\left|\begin{array}{ccc}A & D & E \\ G & B & F \\ I & J & C\end{array}\right|>0$

\section{OBSERVATION AND PROPOSITION}

Our objectives in this section are to analyze the structure of our model in order to gain insights into its behaviour corresponding to reliability, demand rate and production lot size. For the purpose of brevity, all the proofs are omitted for the following propositions.

\section{Proposition 1}

If $r<.5, f_{r}$ turns to be positive; which means that the curve of total average cost $M_{\tilde{T C}}(r, \lambda, q)$ is a rising one from left to right.

\section{Proposition 2}

The curve of total average cost $M_{\tilde{T C}}(r, \lambda, q)$ is concave upward in $r, \lambda$ and $q$.

\section{Proposition 3}

The total average cost $M_{\tilde{T C}}(r, \lambda, q)$ is an increasing function with respect to $\mathrm{H}$ and decreasing function with respect to both $\lambda$ and $q$.

\section{Numerical Analysis}

Here the following numerical examples are considered to illustrate the effectiveness of the proposed model.

\section{Example - 1}

$\mathrm{a}=10, \mathrm{~b}=1, \mathrm{c}=1, \mathrm{H}=5, \mathrm{~S}=100, \quad \Delta_{1}=0.1, \quad \Delta_{2}=0.2, \quad \Delta_{3}=$ 0.01 and $\Delta_{4}=0.02$.

Table 2. Crisp and Fuzzy Values with Relative Error

\begin{tabular}{|c|c|c|c|c|c|}
\hline $\mathbf{H}$ & 5 & 10 & 15 & 20 & 25 \\
\hline$r_{1}$ & 0.5 & 0.5 & 0.5 & 0.5 & 0.5 \\
\hline$\lambda_{l}$ & 0.464158883 & 0.368403149 & 0.321829794 & 0.292401773 & 0.271441761 \\
\hline$q_{1}$ & 4.641588835 & 3.684031503 & 3.218297953 & 2.924017738 & 2.714417617 \\
\hline$Z_{1}(r, \lambda, q)$ & 58.01986047 & 92.10078765 & 120.6861733 & 146.2008872 & 169.6511013 \\
\hline $\begin{array}{ccc}A & D & E \\
G & B & F \\
I & J & C\end{array} \mid>0$. & 742324.5439 & 3803972.985 & 9527967.299 & 18238743.29 & 30248694.97 \\
\hline$r^{*}$ & 0.5 & 0.5 & 0.5 & 0.5 & 0.5 \\
\hline$\lambda^{*}$ & 0.464004269 & 0.368321673 & 0.321770211 & 0.292353051 & 0.271399846 \\
\hline$q^{*}$ & 4.64158883 & 3.684438951 & 3.218774674 & 2.92450502 & 2.71490013 \\
\hline$M_{\tilde{T C}}^{*}(r, \lambda, q)$ & 58.0585379 & 92.14164968 & 120.7308732 & 146.2496219 & 169.7038824 \\
\hline $\begin{array}{ccc}A & D & E \\
G & B & F \\
I & J & C\end{array} \mid>0$ & 744153.8235 & 3810034.092 & 9540793.474 & 18261096.16 & 30283571.66 \\
\hline R. E. & $6.67 \times 10^{-2}$ & $4.44 \times 10^{-2}$ & $3.70 \times 10^{-2}$ & $3.33 \times 10^{-2}$ & $3.11 \times 10^{-2}$ \\
\hline
\end{tabular}



Example - 2
$\mathrm{a}=10, \mathrm{~b}=1, \mathrm{c}=1, \mathrm{H}=10, \mathrm{~S}=100, \Delta_{1}=0.1, \Delta_{2}=0.2, \Delta_{3}=$ 0.01 and $\Delta_{4}=0.02$.
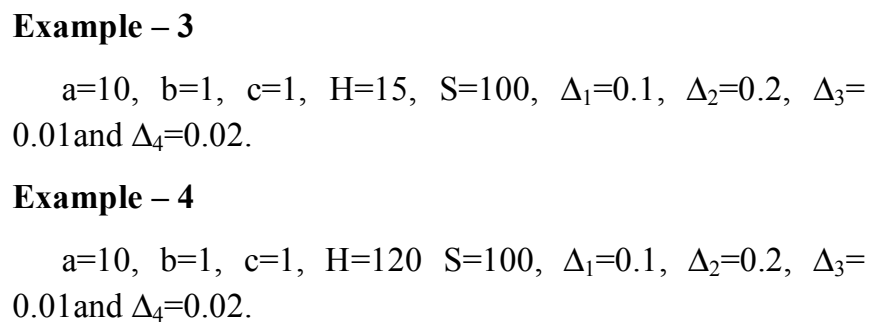

Example - 5

$\mathrm{a}=10, \mathrm{~b}=1, \mathrm{c}=1, \mathrm{H}=25, \mathrm{~S}=100, \Delta_{1}=0.1, \Delta_{2}=0.2, \Delta_{3}=$ 0.01 and $\Delta_{4}=0.02$.

Table 2 presents' five sets of solutions and obtains the computing results for each case as follows:

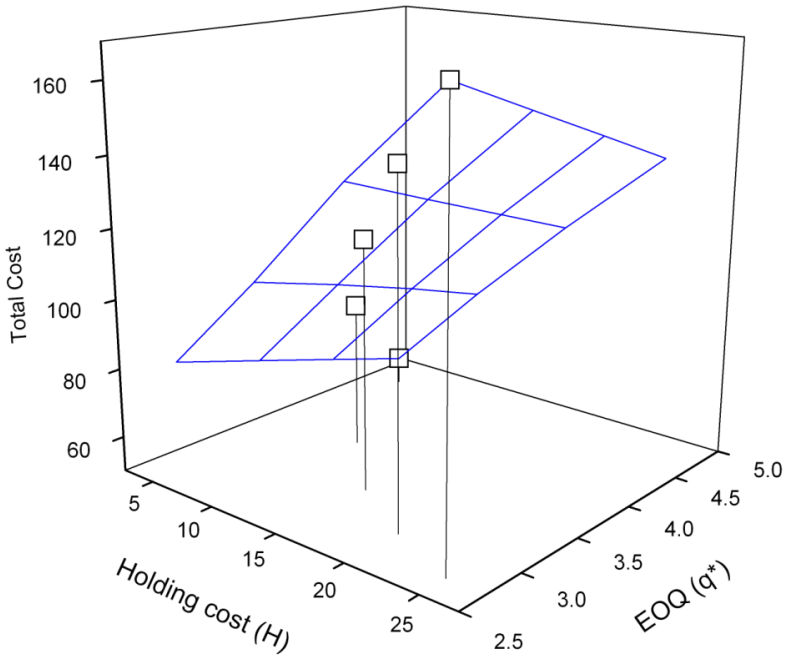

Fig. (1). The Behaviour of Total Cost to the Holding Cost and Changing Lot Size.

\section{SENSITIVITY ANALYSIS}

In Tables 3.1 to $\mathbf{3 . 5}$, it can be seen that the demand $\lambda$, the optimal order quantity $q$ and the optimal total average cost $M_{\tilde{T C}}$ are insensitive to the change of the parameters $\Delta_{1}, \Delta_{2}$, $\Delta_{3}$ and $\Delta_{4}$. The demand $\lambda$, the optimum order quantity $q$ and the optimum total average cost $M_{\tilde{T C}}$ are very sensitive to the changes of the parameters $a, b$ and $c$. This is a very practical aspect of the proposed fuzzy model.

The above mentioned tables illustrate the relative changes of $\lambda$, order quantity $q$ and total cost $M_{\tilde{T C}}$, when each parameter is being changed from $-60 \%$ to $+60 \%$. For all cases it also shows that the $60 \%$ decrease in parameter $b$ gives a minimum optimal total average cost.

In the Tables $\mathbf{3 . 1}$ to $\mathbf{3 . 5}$,

Relative error $\frac{\left(\lambda_{*}-\lambda^{*}\right)}{\lambda^{*}} \times 100 \%=\bar{\lambda}_{*} \%$; $\frac{\left(q_{*}-q^{*}\right)}{q^{*}} \times 100 \%=\bar{q}_{*} \%$ and $\frac{M_{\tilde{T C}}-M_{\tilde{T C}^{*}}}{M_{\tilde{T C}^{*}}} \times 100 \%=\bar{M}_{\tilde{T C_{*}}} \%$

\section{EFFECTIVENESS OF THE TOTAL AVERAGE COST}

We focus on the analysis of effectiveness of cost estimate in Table $\mathbf{4}$ which are studied in comparison. This estimates which type of relationship on decision parameters play the superior role in respect to the relative performance.

The sensitivity analysis $M_{\tilde{T C}_{i}}(i=1,2,3,4)$ is the minimum total cost of the decision parameter $\Delta_{\mathrm{i}}$ and $M_{\tilde{T C_{i}}}^{*}$ is the optimum total cost for $(i=1,2,3,4)$.

\section{ROBUST}

In the previous section it is observed that, the best order size $q^{*}$ comes out to be the solution of the equation (18). In practice, however, the exact form of the decision parameters characterized by the numerical values of the parameters $\Delta_{1}$, $\Delta_{2}, \Delta_{3}$ and $\Delta_{4}$, that suitably represent variations in the proportion of the units in a particular situation may not be known in advance. The possible non availability of such records and the errors of estimates must however, be kept in mind. Hence it will be worthwhile to investigate how the best order size $q^{*}$ changes with the change in any or more of the four parameters $\Delta_{1}, \Delta_{2}, \Delta_{3}$ and $\Delta_{4}$ of the given functional values of the best order size $q^{*}$ for different values of $\Delta_{1}, \Delta_{2}$, $\Delta_{3}$ and $\Delta_{4}$ that are shown in Tables 5.1 to 5.9. Tables 5.1(a) to 5.9(a) summarise the values of the ratio $q^{\prime *}$ for several values of decision parameters, $q^{\prime}$ is the changing value of $q^{*}$.

\section{INTERPRETATION OF THE ANALYSES}

Tables 5.1 and 5.2 summarize the results of an analysis where for a fixed value of $\Delta_{2}^{\prime}$ or $\Delta_{1}^{\prime}$, the best order size is a decreasing function of $\Delta_{4}^{\prime}$, while it is an increasing or decreasing function of $\Delta_{2}^{\prime}$ or $\Delta_{1}^{\prime}$ respectively for a fixed $\Delta_{4}^{\prime}$.

Combining the results of Tables $\mathbf{5 . 3}$ and 5.4, for a fixed value of $\Delta_{2}^{\prime}$ or $\Delta_{1}^{\prime}$, the best order size is an increasing function of $\Delta_{3}^{\prime}$, and for alternate case it is an increasing or decreasing function of $\Delta_{2}^{\prime}$ or $\Delta_{1}^{\prime}$ respectively for a fixed $\Delta_{3}^{\prime}$. Of course, the sensitivity analysis focused on changes in one decision parameter at a time.

But when several parameters are favourable to this strategy, the gains offered on this strategy remain modest. Tables $\mathbf{5 . 5}$ and $\mathbf{5 . 6}$ show for fixed values of $\Delta_{1}^{\prime}$ and $\Delta_{2}^{\prime}$, the best order size is an increasing function of $\Delta_{3}^{\prime}$ or $\Delta_{4}^{\prime}$, while it is a decreasing function of $\Delta_{1}^{\prime}$ and $\Delta_{2}^{\prime}$ for a fixed $\Delta_{3}^{\prime}$ or $\Delta_{4}^{\prime}$. Tables $\mathbf{5 . 7}$ and $\mathbf{5 . 8}$ indicate that, for a fixed value of $\Delta_{2}^{\prime}$ 
Table 3.1. Sensitivity Analysis for $\mathbf{H = 5}$

\begin{tabular}{|c|c|c|c|c|c|c|c|}
\hline Parameter & $\%$ Change & $\lambda *$ & $\mathbf{q}$ & $\boldsymbol{M}_{\tau \tilde{T}}$ & $\bar{\lambda} \%$ & $\bar{q}_{*} \%$ & $\overline{\boldsymbol{M}}_{\tilde{r c}} \%$ \\
\hline \multirow[t]{6}{*}{ a } & +60 & 0.330082713 & 5.503212257 & 68.83601303 & -28.86213877 & 18.56311402 & 18.5631184 \\
\hline & +40 & 0.251900252 & 6.299605492 & 78.79756527 & -45.71165206 & 35.72088616 & 35.72089157 \\
\hline & +20 & 0.158687215 & 7.937005563 & 99.27871122 & -30.5317054 & 70.99760133 & 70.99760829 \\
\hline & -20 & 0.158687215 & 7.937005561 & 59.5672266 & -30.5317054 & 70.9976133 & 2.59856475 \\
\hline & -40 & 0.251900252 & 6.299605509 & 47.27853893 & -45.71165206 & 35.72088653 & -18.56746546 \\
\hline & -60 & 0.330082713 & 5.503212267 & 41.30160809 & -28.86213877 & 18.56311424 & -28.86212849 \\
\hline \multirow[t]{6}{*}{$\mathrm{b}$} & +60 & 0.286521478 & 5.906756443 & 77.57786199 & -38.25024959 & 27.25720996 & 33.62007518 \\
\hline & +40 & 0.187106962 & 7.309416011 & 99.26535006 & -59.67559471 & 57.4765943 & 70.97459504 \\
\hline & +20 & 0.092998806 & 10.36785629 & 146.9758825 & -37.1005463 & 123.3686927 & 153.151195 \\
\hline & -20 & 0.00101424532 & 99.27871537 & 1614.354639 & -99.78141466 & 2038.895085 & 2680.536716 \\
\hline & -40 & $5.87545 \times 10^{-11}$ & 412483.9939 & 7567248.203 & -99.99999999 & 8886598.262 & 13033724.96 \\
\hline & -60 & $9.81456 \times 10^{+11}$ & $3.1914808 \times 10^{-6}$ & $7.185618956 \times 10^{-5}$ & $2.115187436 \times 10^{14}$ & -99.99993124 & -99.99987623 \\
\hline \multirow[t]{6}{*}{$\mathrm{c}$} & +60 & 0.558208708 & 4.231841088 & 52.93327875 & 20.30249403 & -8.827747502 & -8.827744093 \\
\hline & +40 & 0.612257256 & 4.040737928 & 50.5428967 & 31.95078082 & -12.94494028 & -12.94493708 \\
\hline & +20 & 0.671539053 & 3.858264682 & 48.26046056 & 44.72691263 & -16.87620422 & -16.87620408 \\
\hline & -20 & 0.807878292 & 3.51766667 & 43.99789423 & 74.11009897 & -24.21416892 & -24.21804644 \\
\hline & -40 & 0.886101093 & 3.358814497 & 42.01317113 & 90.96830616 & -27.63653525 & -27.63653263 \\
\hline & -60 & 0.971897811 & 3.207135816 & 40.11592379 & 109.458808 & -30.90435337 & -30.90435061 \\
\hline \multirow[t]{6}{*}{$\Delta_{1}$} & +60 & 0.463983618 & 4.642001448 & 58.0637014 & -0.004450605 & 0.008889585 & 0.00889361 \\
\hline & +40 & 0.63973313 & 4.642207674 & 58.06628073 & -0.00667149 & 0.013332589 & 0.013336246 \\
\hline & +20 & 0.463963008 & 4.64241387 & 58.06886009 & -0.008892375 & 0.017774947 & 0.017778935 \\
\hline & -20 & 0.463942402 & 4.642826285 & 58.0740185 & -0.013333282 & 0.026660159 & 0.026663778 \\
\hline & -40 & 0.4639321 & 4.643032466 & 58.07659773 & -0.01555352 & 0.031765329 & 0.031106243 \\
\hline & -60 & 0.463921799 & 4.64323867 & 58.07917678 & -0.017773543 & 0.035544725 & 0.035548397 \\
\hline \multirow[t]{6}{*}{$\Delta_{2}$} & +60 & 0.464045471 & 4.640764087 & 58.04822388 & 0.008879659 & -0.017768549 & -0.017764863 \\
\hline & +40 & 0.464066095 & 4.640351597 & 58.04306429 & 0.013324446 & -0.026655376 & -0.026651739 \\
\hline & +20 & 0.464086724 & 4.639939067 & 58.03790443 & 0.01777031 & -0.035543066 & -0.035539079 \\
\hline & -20 & 0.464127991 & 4.639113991 & 58.02758415 & 0.026663978 & -0.053318789 & -0.053314725 \\
\hline & -40 & 0.464148631 & 4.638701421 & 58.02242341 & 0.031112213 & -0.062207341 & -0.062203581 \\
\hline & -60 & 0.464045471 & 4.63890752 & 58.02964595 & 0.008879659 & -0.057767072 & -0.049763481 \\
\hline \multirow[t]{6}{*}{$\Delta_{3}$} & +60 & 0.463963022 & 4.641176769 & 58.06885665 & -0.008889357 & -0.008866166 & 0.01777301 \\
\hline & +40 & 0.463942423 & 4.640970703 & 58.07401338 & -0.013328756 & -0.013317142 & 0.026654959 \\
\hline & +20 & 0.463921827 & 4.64076476 & 58.07916986 & -0.017767508 & -0.017755859 & 0.035536478 \\
\hline & -20 & 0.463880646 & 4.640352726 & 58.08948223 & -0.026642642 & -0.026631053 & 0.053298500 \\
\hline & -40 & 0.463860061 & 4.63976025 & 58.09463828 & -0.031079024 & -0.039395561 & 0.062179278 \\
\hline & -60 & 0.46383948 & 4.639554385 & 58.09979384 & -0.035514543 & -0.043830788 & 0.071059212 \\
\hline \multirow[t]{6}{*}{$\Delta_{4}$} & +60 & 0.464086697 & 4.642027162 & 58.03791138 & 0.017764491 & 0.009443576 & -0.035527108 \\
\hline & +40 & 0.46412795 & 4.64243981 & 58.02759457 & 0.026655142 & 0.018333808 & -0.053296777 \\
\hline & +20 & 0.464169219 & 4.642852598 & 58.0172767 & 0.035549241 & 0.027227056 & -0.071068272 \\
\hline & -20 & 0.4642518 & 4.64367862 & 57.99663829 & 0.053346707 & 0.04502316 & -0.10661586 \\
\hline & -40 & 0.464293113 & 4.644091849 & 57.9821887 & 0.062250289 & 0.053925909 & -0.131503828 \\
\hline & -60 & 0.46433444 & 4.644505229 & 57.97599616 & 0.071156888 & 0.062831911 & -0.142169856 \\
\hline
\end{tabular}


Table 3.2. Sensitivity Analysis for $H=10$

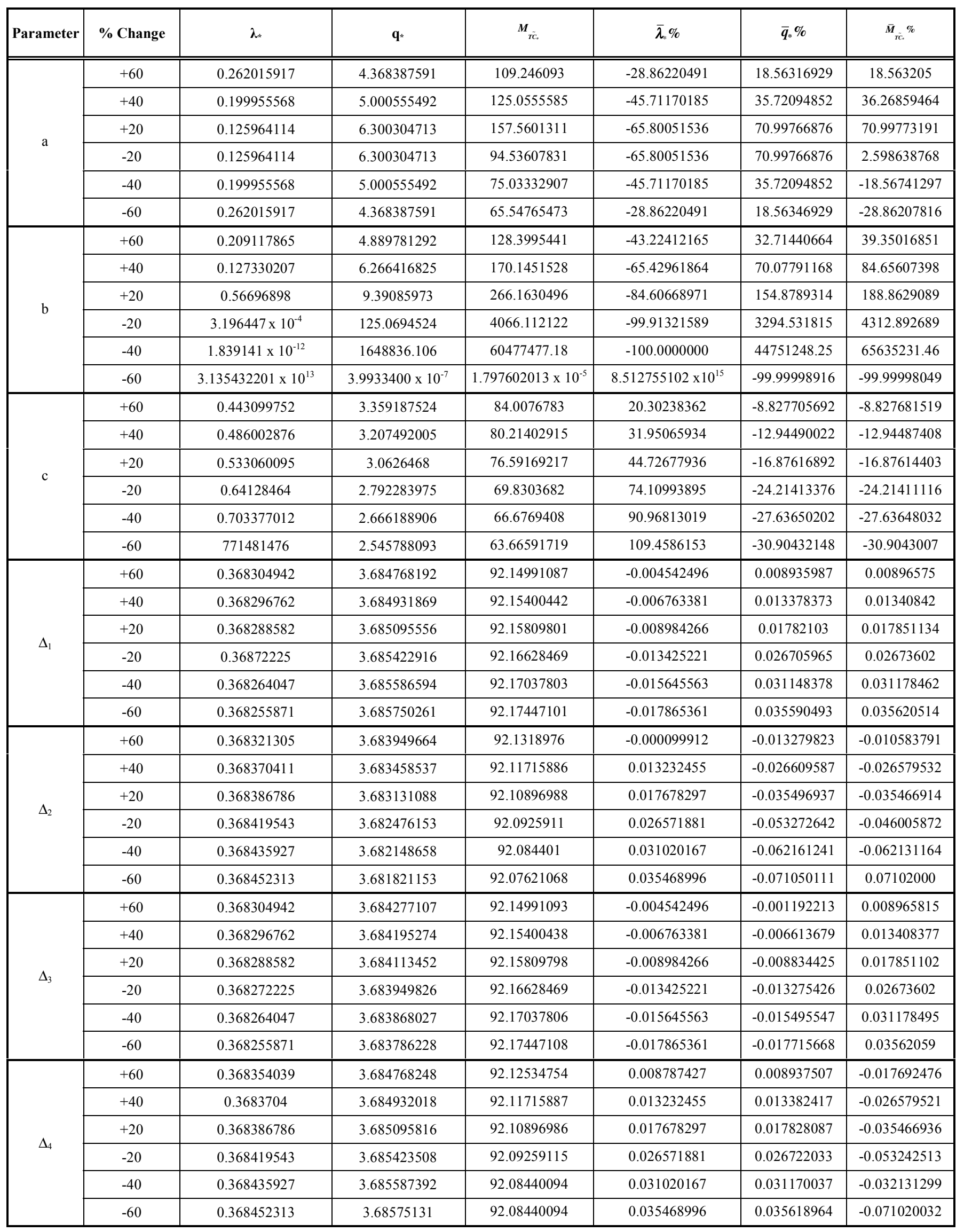


Table 3.3. Sensitivity Analysis for $H=15$

\begin{tabular}{|c|c|c|c|c|c|c|c|}
\hline Parameter & $\%$ Change & $\lambda *$ & $\mathbf{q} *$ & $M_{\tilde{T C} .}$ & $\bar{\lambda}_{*} \%$ & $\bar{q}_{*} \%$ & $\bar{M}_{\tilde{T} \tilde{C}_{i}} \%$ \\
\hline \multirow{6}{*}{$\mathrm{a}$} & +60 & 0.228900461 & 3.816279371 & 143.1422788 & -28.86213416 & 18.56311042 & 18.56311067 \\
\hline & +40 & 0.174683745 & 4.368549356 & 163.8570054 & -45.71164793 & 35.72088134 & 35.72088138 \\
\hline & +20 & 0.110043864 & 5.504027285 & 206.4468898 & -65.80048114 & 70.99759512 & 70.99759451 \\
\hline & -20 & 0.110043864 & 5.504027285 & 123.8681337 & -65.80048114 & 70.99759512 & 2.598556953 \\
\hline & -40 & 0.174633745 & 4.368549356 & 98.31420288 & -45.71164793 & 35.72088134 & -18.56747144 \\
\hline & -60 & 0.228900461 & 3.816279371 & 85.88536705 & -28.86213416 & 18.56311042 & -28.86213383 \\
\hline \multirow{6}{*}{$\mathrm{b}$} & +60 & 0.173928832 & 4.37801961 & 172.4228301 & -45.94626039 & 36.01510057 & 42.81585599 \\
\hline & +40 & 0.10165524 & 5.7266232 & 233.2071849 & -68.40750432 & 77.91314335 & 93.16284122 \\
\hline & +20 & 0.042443803 & 8.862502044 & 376.7400381 & -86.80928142 & 175.3377587 & 212.0494602 \\
\hline & -20 & $1.62653150 \times 10^{-4}$ & 143.1634825 & 6980.770708 & -99.94945053 & 4347.763419 & 5682.092453 \\
\hline & -40 & $2.423260 \times 10^{-13}$ & 3706555.668 & 204043484.6 & -100.0000000 & 115154144.8 & 169006782.2 \\
\hline & -60 & $2.379646 \times 10^{14}$ & $1.18360643 \times 10^{-7}$ & $7.991118602 \times 10^{-6}$ & $7.39548261 \times 10^{16}$ & -99.99999632 & -99.99999338 \\
\hline \multirow{6}{*}{$\mathrm{c}$} & +60 & 0.387097619 & 2.934629259 & 110.0730525 & 20.30250339 & -8.827751047 & -8.827750862 \\
\hline & +40 & 0.424578339 & 2.802106107 & 105.1031544 & 31.95079112 & -12.94494363 & -12.94426056 \\
\hline & +20 & 0.465688127 & 2.798451114 & 100.4370351 & 44.72692346 & -13.05849594 & -16.80915375 \\
\hline & -20 & 0.560234476 & 2.439375052 & 91.49689258 & 74.11011239 & -24.21417157 & -24.21417144 \\
\hline & -40 & 0.614479168 & 2.329216786 & 87.36503957 & 90.96832056 & -27.63653806 & -27.63653804 \\
\hline & -60 & 0.673976101 & 2.224033094 & 83.4197745 & 109.4588243 & -30.90435587 & -30.90435587 \\
\hline \multirow{6}{*}{$\Delta_{1}$} & +60 & 0.321755916 & 3.219060688 & 120.7416013 & -0.004442611 & 0.008885803 & 0.008885962 \\
\hline & +40 & 0.321748769 & 3.219203692 & 120.7469652 & -0.006663761 & 0.013328612 & 0.013328819 \\
\hline & +20 & 0.321741623 & 3.219346691 & 120.7523288 & -0.008884601 & 0.017771265 & 0.017771427 \\
\hline & -20 & 0.321727333 & 3.219632678 & 120.7630557 & -0.013325658 & 0.02665623 & 0.026656396 \\
\hline & -40 & 0.32172019 & 3.219775661 & 120.7684188 & -0.015545565 & 0.031098386 & 0.03109859 \\
\hline & -60 & 0.321713046 & 3.21991865 & 120.7737821 & -0.017765783 & 0.035540729 & 0.03554095 \\
\hline \multirow{6}{*}{$\Delta_{2}$} & +60 & 0.321798808 & 3.218202616 & 120.7094164 & 0.008887398 & -0.017772539 & -0.017772421 \\
\hline & +40 & 0.32181311 & 3.217916569 & 120.6986873 & 0.013332185 & -0.026659368 & -0.026659212 \\
\hline & +20 & 0.321827415 & 3.217630507 & 120.6879576 & 0.017777904 & -0.035546663 & -0.0355465 \\
\hline & -20 & 0.321856033 & 3.217201375 & 120.6664968 & 0.026671828 & -0.048878817 & -0.053322235 \\
\hline & -40 & 0.321870346 & 3.21677224 & 120.6557654 & 0.031120034 & -0.062211064 & -0.06221093 \\
\hline & -60 & 0.321884661 & 3.216486128 & 120.6450339 & 0.035568861 & -0.071099913 & -0.071099709 \\
\hline \multirow{6}{*}{$\Delta_{3}$} & +60 & 0.321760679 & 3.218679335 & 120.7380263 & -0.002962362 & -0.002961965 & 0.00592483 \\
\hline & +40 & 0.321755914 & 3.218631666 & 120.7416026 & -0.004443232 & -0.004442932 & 0.008887039 \\
\hline & +20 & 0.321751149 & 3.218584001 & 120.7451788 & -0.005924103 & -0.005923775 & 0.011849164 \\
\hline & -20 & 0.32174162 & 3.218488679 & 120.7523312 & -0.008885533 & -0.008885213 & 0.017773415 \\
\hline & -40 & 0.321736856 & 3.218417195 & 120.7559072 & -0.010366093 & -0.011106058 & 0.020735375 \\
\hline & -60 & 0.321732092 & 3.21839337 & 120.7594833 & -0.011846652 & -0.011846247 & 0.023697418 \\
\hline \multirow{6}{*}{$\Delta_{4}$} & +60 & 0.321789276 & 3.218965402 & 120.7165468 & 0.005925035 & 0.005925484 & -0.011866393 \\
\hline & +40 & 0.321798811 & 3.219060778 & 120.7094141 & 0.00888833 & 0.008888599 & -0.017774326 \\
\hline & +20 & 0.321808347 & 3.219156167 & 120.7022605 & 0.01151936 & 0.011852118 & -0.023699571 \\
\hline & -20 & 0.321827422 & 3.219346979 & 120.6879527 & 0.017780079 & 0.017780213 & -0.035550558 \\
\hline & -40 & 0.321836961 & 3.219442403 & 120.6807985 & 0.020744617 & 0.020744816 & -0.0414763 \\
\hline & -60 & 0.321846501 & 3.219537839 & 120.6736442 & 0.023709466 & 0.023709798 & -0.047402125 \\
\hline
\end{tabular}


Table 3.4. Sensitivity Analysis for $\mathbf{H}=\mathbf{2 0}$

\begin{tabular}{|c|c|c|c|c|c|c|c|}
\hline Parameter & $\%$ Change & $\lambda_{*}$ & $\mathbf{q}^{*}$ & $\boldsymbol{M}_{T \tilde{c}_{*}}$ & $\bar{\lambda} \%$ & $\overline{\boldsymbol{q}}_{*} \%$ & $\bar{M}_{\tilde{T} \tilde{c}_{i}} \%$ \\
\hline \multirow{6}{*}{$\mathrm{a}$} & +60 & 0.207973722 & 3.467384111 & 173.3981002 & -28.86213389 & 180.56311025 & 18.56311008 \\
\hline & +40 & 0.158713654 & 3.969163978 & 158.7930202 & -45.7116478 & 35.720881 & 8.576704772 \\
\hline & +20 & 0.099983337 & 5.000833246 & 250.0833357 & -65.80048108 & 70.99759487 & 70.9975947 \\
\hline & -20 & 0.099983337 & 5.000833246 & 150.0500014 & -65.80048108 & 70.99759487 & 2.598556804 \\
\hline & -40 & 0.158713654 & 3.969163978 & 119.094765 & -45.7116478 & 35.720881 & -18.56747152 \\
\hline & -60 & 0.207973722 & 3.467384111 & 104.0388601 & -28.86213389 & 18.56311025 & -28.86213397 \\
\hline \multirow{6}{*}{$\mathrm{b}$} & +60 & 0.152613239 & 4.047716449 & 212.5405312 & -47.79830808 & 38.4068901 & 45.32723465 \\
\hline & +40 & 0.086642856 & 5.372047042 & 291.6740152 & -70.36232176 & 83.69081281 & 99.43573967 \\
\hline & +20 & 0.034561259 & 8.505726406 & 482.0714975 & -88.17824583 & 190.8432828 & 229.6223889 \\
\hline & -20 & $1.0070973 \times 10^{-4}$ & 157.5688882 & 10243.6848 & -99.96555202 & 5287.882295 & 6904.247031 \\
\hline & -40 & $5.7521076 \times 10^{-14}$ & 6593145.89 & 483577948.4 & -100.000000 & 225444747.8 & 330652343.5 \\
\hline & -60 & $1.0025027 \times 10^{15}$ & $4.994171025 \times 10^{-8}$ & $4.495503022 \times 10^{-6}$ & $3.429082394 \times 10^{-17}$ & -99.99999829 & -99.99999693 \\
\hline \multirow{6}{*}{$\mathrm{c}$} & +60 & 0.35170804 & 2.666336992 & 133.339069 & 20.3025037 & -8.827751234 & -8.827751301 \\
\hline & +40 & 0.385762163 & 2.545929494 & 127.3176908 & 31.95079089 & -12.94494362 & -12.94494362 \\
\hline & +20 & 0.423113577 & 2.430959397 & 121.568228 & 44.72692368 & -16.87621049 & -16.8762104 \\
\hline & -20 & 0.509016226 & 2.216360353 & 110.8364874 & 74.1101125 & -24.2141717 & -24.21417193 \\
\hline & -40 & 0.558301712 & 2.116273075 & 105.8312892 & 90.9683207 & -27.63653813 & -27.63653839 \\
\hline & -60 & 0.612359264 & 2.020705578 & 101.052118 & 109.4588245 & -30.90435598 & -30.90435607 \\
\hline \multirow{6}{*}{$\Delta_{1}$} & +60 & 0.292340063 & 2.924764884 & 146.2626172 & -0.004442573 & 0.008885742 & 0.008885698 \\
\hline & +40 & 0.292333569 & 2.924894816 & 146.269115 & -0.00666386 & 0.13328614 & 0.013328649 \\
\hline & +20 & 0.292327077 & 2.925024738 & 146.2756121 & -0.008884463 & 0.017771144 & 0.017771122 \\
\hline & -20 & 0.292314094 & 2.925284577 & 146.2938042 & -0.013325326 & 0.026656032 & 0.030210197 \\
\hline & -40 & 0.292307603 & 2.925414494 & 146.2951031 & -0.015545587 & 0.03109839 & 0.031098336 \\
\hline & -60 & 0.292301112 & 2.92554441 & 146.3016001 & -0.017765848 & 0.035540715 & 0.035540741 \\
\hline \multirow{6}{*}{$\Delta_{2}$} & +60 & 0.292379033 & 2.923985263 & 146.2236298 & 0.008887199 & -0.017772477 & -0.017772422 \\
\hline & +40 & 0.292392028 & 2.923725365 & 146.2106327 & 0.013332168 & -0.026659383 & -0.026659351 \\
\hline & +20 & 0.292405026 & 2.923465451 & 146.1976347 & 0.017778162 & -0.035546835 & -0.035546895 \\
\hline & -20 & 0.292431027 & 2.922945599 & 146.1716378 & 0.026671861 & -0.053322561 & -0.053322599 \\
\hline & -40 & 0.292444031 & 2.922685655 & 146.1586385 & 0.031119907 & -0.06221104 & -0.062211032 \\
\hline & -60 & 0.292457038 & 2.922425696 & 146.1456383 & 0.03556898 & -0.071100031 & -0.071100081 \\
\hline \multirow{6}{*}{$\Delta_{3}$} & +60 & 0.292346555 & 2.924440048 & 146.2561209 & -0.00222197 & -0.00222164 & 0.004443772 \\
\hline & +40 & 0.292343308 & 2.92440756 & 146.2593701 & -0.003332614 & -0.003332529 & 0.006665453 \\
\hline & +20 & 0.29234006 & 2.924375079 & 146.2626197 & -0.004443599 & -0.004443179 & 0.008887407 \\
\hline & -20 & 0.292333566 & 2.924310115 & 146.2691182 & -0.006664886 & -0.006664546 & 0.013330837 \\
\hline & -40 & 0.292330319 & 2.924277636 & 146.2723674 & -0.00777553 & -0.007775127 & 0.015552518 \\
\hline & -60 & 0.292327073 & 2.924245154 & 146.2756163 & -0.008885831 & -0.008885811 & 0.017773994 \\
\hline \multirow{6}{*}{$\Delta_{4}$} & +60 & 0.292366043 & 2.92463499 & 146.2366239 & 0.004443941 & 0.00444417 & -0.008887544 \\
\hline & +40 & 0.29237254 & 2.924699983 & 146.2301247 & 0.006666255 & 0.006666529 & -0.013331453 \\
\hline & +20 & 0.292379038 & 2.92476498 & 146.2236252 & 0.00888891 & 0.008889025 & -0.017775567 \\
\hline & -20 & 0.292392035 & 2.924894995 & 146.210626 & 0.013334562 & 0.013334735 & -0.026663932 \\
\hline & -40 & 0.292398534 & 2.924960012 & 146.2041263 & 0.015557559 & 0.015557914 & -0.031108182 \\
\hline & -60 & 0.292405034 & 2.925025034 & 146.1976263 & 0.017780898 & 0.017781265 & -0.035552638 \\
\hline
\end{tabular}


Table 3.5. Sensitivity Analysis for $\mathbf{H}=\mathbf{2 5}$

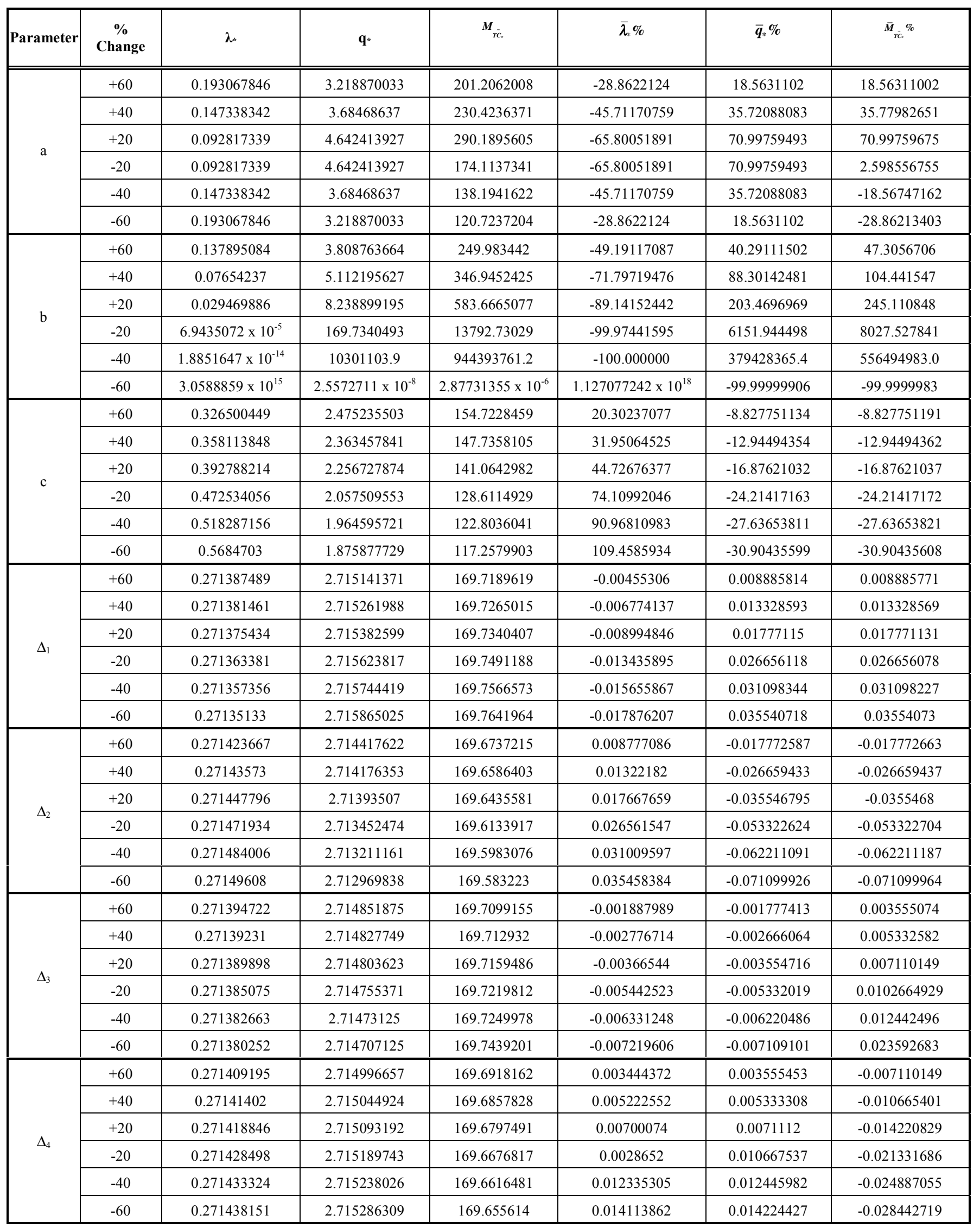


Table 4.

\begin{tabular}{|c|c|c|c|c|c|c|}
\hline Conditions & $M_{\tilde{T C}}$ & $\boldsymbol{M}_{\tilde{T C}}^{*}$ for $\mathbf{H = 5}$ & $\begin{array}{c}M_{\tilde{T C}}^{*} \\
\text { for } \mathbf{H = 1 0}\end{array}$ & 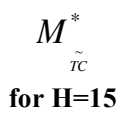 & $\begin{array}{c}M_{\tilde{T C}}^{*} \\
\text { for } \mathbf{H}=\mathbf{2 0}\end{array}$ & $M_{\tilde{T C}}^{*}$ \\
\hline$\Delta_{1}<\Delta_{2}$ & $\operatorname{Min}\left\{M_{\tilde{T C_{1}}}, M_{\tau \tilde{C}_{2}}\right\}$ & $M_{\tilde{T \tilde{C}_{2}}}$ & $M_{\tilde{T C_{2}}}$ & $M_{\tilde{T C_{2}}}$ & $M_{\tilde{T C_{2}}}$ & $M_{\tilde{T_{2}}}$ \\
\hline$\Delta_{1}>\Delta_{2}$ & $\operatorname{Min}\left\{M_{\tilde{T \tilde{C}_{1}}}, M_{\tilde{T C_{2}}}\right\}$ & $M_{\tilde{T \tilde{C}_{2}}}$ & $M_{\tilde{T C_{2}}}$ & $M_{\tilde{\tilde{T}_{2}}}$ & $M_{\tilde{T C_{2}}}$ & $M_{\tilde{T \tilde{C}_{2}}}$ \\
\hline$\Delta_{1}=\Delta_{2}$ & $\operatorname{Min}\left\{M_{\tilde{T \tilde{C}_{1}}}, M_{\tilde{T \tilde{C}_{2}}}\right\}$ & $M_{\tilde{T \tilde{C}_{2}}}$ & $M_{\tilde{T \tilde{C}_{2}}}$ & $M_{\tilde{T \tilde{C}_{2}}}$ & $M_{\tilde{T \tilde{C}_{2}}}$ & $M_{\tilde{T \tilde{C}_{2}}}$ \\
\hline$\Delta_{3}<\Delta_{4}$ & $\operatorname{Min}\left\{M_{\tilde{T \tilde{C}_{3}}}, M_{\tilde{T \tilde{C}_{4}}}\right\}$ & $M_{\tilde{T \tilde{C}_{4}}}$ & $M_{\tilde{T \tilde{C}_{4}}}$ & $M_{\tilde{\tilde{C}_{4}}}$ & $M_{\tilde{T \tilde{C}_{4}}}$ & $M_{\tilde{T \tilde{C}_{4}}}$ \\
\hline$\Delta_{3}>\Delta_{4}$ & $\operatorname{Min}\left\{M_{\tilde{T \tilde{C}_{3}}}, M_{\tilde{T \tilde{C}_{4}}}\right\}$ & $M_{\tilde{T \tilde{C}_{4}}}$ & $M_{\tilde{T \tilde{C}_{4}}}$ & $M_{\tilde{T \tilde{C}_{4}}}$ & $M_{\tilde{T \tilde{C}_{4}}}$ & $M_{\tilde{T C_{4}}}$ \\
\hline$\Delta_{3}=\Delta_{4}$ & $\operatorname{Min}\left\{M_{\tilde{T \tilde{C}_{3}}}, M_{\tilde{T \tilde{C}_{4}}}\right\}$ & $M_{\tilde{T \tilde{C}_{4}}}$ & $M_{\tilde{T \tilde{C}_{4}}}$ & $M_{\tilde{T \tilde{C}_{4}}}$ & $M_{\tilde{T \tilde{C}_{4}}}$ & $M_{\tilde{T \tilde{C}_{4}}}$ \\
\hline
\end{tabular}

Table 5.1. Values of the Best Order Quantity $q^{*}(\mathrm{H}=5)$

\begin{tabular}{|c|c|c|c|c|c|c|c|c|}
\hline & \multicolumn{1}{|c|}{$\Delta_{2}^{\prime}$} & $\mathbf{0 . 3}$ & $\mathbf{0 . 4}$ & $\mathbf{0 . 5}$ & $\mathbf{0 . 6}$ & $\mathbf{0 . 7}$ & $\mathbf{0 . 8}$ & $\mathbf{0 . 9}$ \\
\hline \hline$\Delta_{4}^{\prime}$ & & & & & $\mathbf{1 . 0}$ \\
\hline 0.03 & 4.641589525 & 4.642620244 & 4.643650849 & 4.644681338 & 4.645711717 & 4.646741981 & 4.647772129 & 4.648802163 \\
\hline 0.04 & 4.632860263 & 4.641075536 & 4.642620758 & 4.643651020 & 4.644681668 & 4.645711200 & 4.646741122 & 4.647770929 \\
\hline 0.05 & 4.639531168 & 4.640561430 & 4.641591581 & 4.642621613 & 4.643651534 & 4.644681340 & 4.645711030 & 4.646740606 \\
\hline 0.06 & 4.638503357 & 4.639533392 & 4.640563312 & 4.641593117 & 4.642622812 & 4.643652387 & 4.644681851 & 4.645711200 \\
\hline 0.07 & 4.637476460 & 4.638506264 & 4.639535958 & 4.640565537 & 4.641595001 & 4.642624350 & 4.643653583 & 4.644682706 \\
\hline 0.08 & 4.636450471 & 4.637480049 & 4.638509513 & 4.639538861 & 4.640568099 & 4.641597221 & 4.642626229 & 4.643655121 \\
\hline 0.09 & 4.635425388 & 4.636454736 & 4.637483973 & 4.638513095 & 4.639542103 & 4.640570999 & 4.641599781 & 4.642628447 \\
\hline 0.10 & 4.634401209 & 4.635430330 & 4.636459342 & 4.637488239 & 4.638517021 & 4.639545687 & 4.640574238 & 4.641602679 \\
\hline
\end{tabular}

Table 5.1.a. Values of the Ratio $q^{\prime *}(\mathbf{H}=5)$

\begin{tabular}{|c|c|c|c|c|c|c|c|c|}
\hline & $\Delta_{2}^{\prime}$ & $\mathbf{0 . 3}$ & $\mathbf{0 . 4}$ & $\mathbf{0 . 5}$ & $\mathbf{0 . 6}$ & $\mathbf{0 . 7}$ & $\mathbf{0 . 8}$ & $\mathbf{0 . 9}$ \\
\hline \hline$\Delta_{4}^{\prime}$ & & & & & $\mathbf{1 . 0}$ \\
\hline 0.03 & 1.000000150 & 1.000222202 & 1.000444249 & 1.000666261 & 1.000888249 & 1.001110213 & 1.001332151 & 1.001554065 \\
\hline 0.04 & 0.998119487 & 0.999889414 & 1.000222322 & 1.000444285 & 1.000666224 & 1.000888138 & 1.001110028 & 1.001331893 \\
\hline 0.05 & 0.999556690 & 0.999778653 & 1.000000593 & 1.000222506 & 1.000444396 & 1.000666261 & 1.000888101 & 1.001109916 \\
\hline 0.06 & 0.999335255 & 0.999557169 & 0.999779058 & 1.000000924 & 1.000222765 & 1.00044458 & 1.000666371 & 1.000888138 \\
\hline 0.07 & 0.999114016 & 0.999335881 & 0.999557722 & 0.999779538 & 1.000001330 & 1.000223096 & 1.000444838 & 1.0006665555 \\
\hline 0.08 & 0.998892973 & 0.999114790 & 0.999336581 & 0.999558347 & 0.999780090 & 1.000001808 & 1.000223501 & 1.000445169 \\
\hline 0.09 & 0.998672126 & 0.998893893 & 0.999115635 & 0.999337353 & 0.999559046 & 0.999780715 & 1.000002359 & 1.000223979 \\
\hline 0.10 & 0.998451474 & 0.998673191 & 0.998894885 & 0.999116554 & 0.999338198 & 0.999559818 & 0.999781412 & 1.000002984 \\
\hline
\end{tabular}


Table 5.2. Values of the Best Order Quantity $q *(H=10)$

\begin{tabular}{|c|c|c|c|c|c|c|c|c|}
\hline & $\Delta_{2}^{\prime}$ & $\mathbf{0 . 2}$ & $\mathbf{0 . 3}$ & $\mathbf{0 . 4}$ & $\mathbf{0 . 5}$ & $\mathbf{0 . 6}$ & $\mathbf{0 . 7}$ & $\mathbf{0 . 8}$ \\
\hline$\Delta_{4}^{\prime}$ & & & & & $\mathbf{0 . 9}$ \\
\hline \hline 0.03 & 3.683213193 & 3.682394654 & 3.681576025 & 3.680757307 & 3.679938498 & 3.679119594 & 3.678300601 & 3.677481518 \\
\hline 0.04 & 3.682804309 & 3.681985864 & 3.681167323 & 3.680144409 & 3.679529977 & 3.678711166 & 3.677892266 & 3.677073271 \\
\hline 0.05 & 3.682395608 & 3.681577250 & 3.680758808 & 3.679940270 & 3.679121638 & 3.678302920 & 3.677484108 & 3.676665206 \\
\hline 0.06 & 3.681987088 & 3.681168824 & 3.680350469 & 3.679532020 & 3.678713481 & 3.677894852 & 3.677076132 & 3.676257324 \\
\hline 0.07 & 3.681578751 & 3.680760574 & 3.679942308 & 3.679123957 & 3.678305505 & 3.677486969 & 3.676668338 & 3.675849617 \\
\hline 0.08 & 3.68117059 & 3.680352507 & 3.679534333 & 3.678716070 & 3.677897712 & 3.677079263 & 3.676260725 & 3.675442098 \\
\hline 0.09 & 3.680762615 & 3.679944625 & 3.679126540 & 3.678308364 & 3.677490099 & 3.676671744 & 3.675853294 & 3.684232319 \\
\hline 0.10 & 3.680354822 & 3.679536920 & 3.678718927 & 3.677900840 & 3.677082668 & 3.676264400 & 3.675446043 & 3.674627597 \\
\hline
\end{tabular}

Table 5.2.a. Values of the Ratio $q^{\prime *}(\mathrm{H}=\mathbf{1 0})$

\begin{tabular}{|c|c|c|c|c|c|c|c|c|}
\hline & $\Delta_{2}^{\prime}$ & $\mathbf{0 . 2}$ & $\mathbf{0 . 3}$ & $\mathbf{0 . 4}$ & $\mathbf{0 . 5}$ & $\mathbf{0 . 6}$ & $\mathbf{0 . 7}$ & $\mathbf{0 . 8}$ \\
\hline$\Delta_{4}^{\prime}$ & & & & & $\mathbf{0 . 9}$ \\
\hline \hline 0.03 & 0.999667314 & 0.999445153 & 0.999222968 & 0.999000758 & 0.998778524 & 0.998556264 & 0.998333979 & 0.998111671 \\
\hline 0.04 & 0.999556339 & 0.999334203 & 0.999112041 & 0.998834410 & 0.998667646 & 0.998445411 & 0.998223152 & 0.998000867 \\
\hline 0.05 & 0.999445412 & 0.999223300 & 0.999001166 & 0.998779005 & 0.998556818 & 0.998334609 & 0.998112373 & 0.997890114 \\
\hline 0.06 & 0.999334535 & 0.999112449 & 0.998890338 & 0.998668201 & 0.998446040 & 0.998223854 & 0.998001644 & 0.997779410 \\
\hline 0.07 & 0.999223708 & 0.999001645 & 0.998779558 & 0.998557448 & 0.998335310 & 0.998113150 & 0.997890964 & 0.997668753 \\
\hline 0.08 & 0.999112928 & 0.998890891 & 0.998668829 & 0.998446742 & 0.998224630 & 0.998002494 & 0.997780333 & 0.997558148 \\
\hline 0.09 & 0.999002199 & 0.998780187 & 0.998558149 & 0.998336086 & 0.998114000 & 0.997891888 & 0.997669751 & 0.999943917 \\
\hline 0.10 & 0.998891519 & 0.998669531 & 0.998447518 & 0.998225479 & 0.998003418 & 0.997781330 & 0.997559219 & 0.997337083 \\
\hline
\end{tabular}

Table 5.3. Values of the Best Order Quantity $q *(H=15)$

\begin{tabular}{|c|c|c|c|c|c|c|c|c|}
\hline$\Delta_{3}^{\prime}$ & 0.3 & 0.4 & 0.5 & 0.6 & 0.7 & 0.8 & 0.9 & 1.0 \\
\hline 0.02 & 3.219728149 & 3.220443127 & 3.221158025 & 3.221872847 & 3.222587585 & 3.223302247 & 3.224016830 & 3.224731332 \\
\hline 0.03 & 3.219966680 & 3.220681713 & 3.221396665 & 3.222111538 & 3.222826330 & 3.223541047 & 3.224255679 & 3.224970236 \\
\hline 0.04 & 3.220205287 & 3.220920370 & 3.221635377 & 3.222350299 & 3.223065146 & 3.223779913 & 3.224494605 & 3.225209211 \\
\hline 0.05 & 3.220443961 & 3.221159098 & 3.221874155 & 3.222589137 & 3.223304034 & 3.224018855 & 3.224733597 & 3.225448258 \\
\hline 0.06 & 3.220682705 & 3.221397897 & 3.222113009 & 3.222828040 & 3.223542992 & 3.224257868 & 3.224972660 & 3.225687376 \\
\hline 0.07 & 3.220921520 & 3.221636767 & 3.222351929 & 3.223067015 & 3.223782022 & 3.224496948 & 3.225211794 & 3.225926565 \\
\hline 0.08 & 3.221160412 & 3.221875709 & 3.222590925 & 3.223306062 & 3.224021123 & 3.224736099 & 3.225451000 & 3.226165820 \\
\hline 0.09 & 3.221399370 & 3.222114717 & 3.222829988 & 3.223545179 & 3.224260290 & 3.224975326 & 3.225690277 & 3.226405152 \\
\hline
\end{tabular}

Table 5.3.a. Values of the Ratio $q^{\prime *}(\mathrm{H}=15)$

\begin{tabular}{|c|c|c|c|c|c|c|c|c|}
\hline & $\Delta_{2}^{\prime}$ & $\mathbf{0 . 3}$ & $\mathbf{0 . 4}$ & $\mathbf{0 . 5}$ & $\mathbf{0 . 6}$ & $\mathbf{0 . 7}$ & $\mathbf{0 . 8}$ & $\mathbf{0 . 9}$ \\
\hline \hline$\Delta_{3}^{\prime}$ & & & & & $\mathbf{1 . 0}$ \\
\hline \hline 0.02 & 1.000296223 & 1.000518350 & 1.000740453 & 1.000962532 & 1.001184585 & 1.001406614 & 1.001628619 & 1.001850598 \\
\hline 0.03 & 1.003703290 & 1.000592474 & 1.000814593 & 1.001036688 & 1.001258757 & 1.001480804 & 1.001702823 & 1.00192482 \\
\hline 0.04 & 1.000444459 & 1.000666619 & 1.000888755 & 1.001110865 & 1.001332952 & 1.001555014 & 1.001777052 & 1.001999064 \\
\hline 0.05 & 1.000518609 & 1.000740786 & 1.000962938 & 1.001185067 & 1.001407169 & 1.001629248 & 1.001851302 & 1.002073331 \\
\hline 0.06 & 1.000592782 & 1.000814976 & 1.001037145 & 1.001259289 & 1.001481408 & 1.001703504 & 1.001925573 & 1.002147619 \\
\hline 0.07 & 1.000666976 & 1.000891870 & 1.001111372 & 1.001333533 & 1.001555669 & 1.001777780 & 1.001999867 & 1.002221930 \\
\hline 0.08 & 1.000741194 & 1.000963421 & 1.001185622 & 1.001407799 & 1.001629952 & 1.001852079 & 1.002074182 & 1.002296261 \\
\hline 0.09 & 1.000815433 & 1.001037675 & 1.001259894 & 1.001482087 & 1.001926401 & 1.001926401 & 0.986819404 & 1.002370616 \\
\hline
\end{tabular}


Table 5.4. Values of the Best Order Quantity $q^{*}(\mathrm{H}=\mathbf{2 0})$

\begin{tabular}{|c|c|c|c|c|c|c|c|c|}
\hline$\Delta_{2}^{\prime}$ & 0.2 & 0.3 & 0.4 & 0.5 & 0.6 & 0.7 & 0.8 & 0.9 \\
\hline 0.02 & 2.924017742 & 2.923367921 & 2.922718035 & 2.922068069 & 2.921418037 & 2.920767926 & 2.920117750 & 2.91946750 \\
\hline 0.03 & 2.924180203 & 2.923530349 & 2.922880426 & 2.922230428 & 2.921580354 & 2.920930211 & 2.920279998 & 2.91962971 \\
\hline 0.04 & 2.924342702 & 2.923692811 & 2.923042851 & 2.922392816 & 2.921742710 & 2.921092530 & 2.920442280 & 2.919791955 \\
\hline 0.05 & 2.924505241 & 2.923855313 & 2.923205315 & 2.922555243 & 2.921905101 & 2.921254883 & 2.920604596 & 2.919954239 \\
\hline 0.06 & 2.924667813 & 2.924017849 & 2.923611621 & 2.92271771 & 2.922067530 & 2.921417276 & 2.920766951 & 2.920116557 \\
\hline 0.07 & 2.924830420 & 2.924180419 & 2.923530352 & 2.922880205 & 2.922229994 & 2.921579702 & 2.920929346 & 2.920278910 \\
\hline 0.08 & 2.924993062 & 2.924343028 & 2.923692924 & 2.923042745 & 2.922392492 & 2.921742168 & 2.921091769 & 2.920441301 \\
\hline 0.09 & 2.925155742 & 2.924505671 & 2.923855530 & 2.923205314 & 2.922555029 & 2.921904668 & 2.921254237 & 2.920603732 \\
\hline
\end{tabular}

Table 5.4.a. Values of the Ratio $q^{\prime *}(\mathbf{H = 2 0})$

\begin{tabular}{|c|c|c|c|c|c|c|c|c|}
\hline$\Delta_{3}^{\prime}$ & 0.2 & 0.3 & 0.4 & 0.5 & 0.6 & 0.7 & 0.8 & 0.9 \\
\hline 0.02 & 0.999833381 & 0.999611182 & 0.999388961 & 0.999166713 & 0.998944442 & 0.998722144 & 0.998499824 & 0.998277479 \\
\hline 0.03 & 0.999888932 & 0.999666722 & 0.999444489 & 0.999222230 & 0.998999945 & 0.998777636 & 0.998555303 & 0.998332945 \\
\hline 0.04 & 0.999944497 & 0.999722274 & 0.999500028 & 0.999277756 & 0.999277756 & 0.999055460 & 0.998610794 & 0.998388423 \\
\hline 0.05 & 1.000000076 & 0.99977784 & 0.999555581 & 0.999333296 & 0.999110988 & 0.998888654 & 0.998666296 & 0.998443914 \\
\hline 0.06 & 1.000055665 & 0.999833417 & 0.999694512 & 0.999388850 & 0.999166529 & 0.998944182 & 0.998721811 & 0.998499416 \\
\hline 0.07 & 1.000111267 & 0.999889006 & 0.999666723 & 0.999444413 & 0.999222081 & 0.998999722 & 0.998777340 & 0.998554931 \\
\hline 0.08 & 1.000166880 & 0.999944608 & 0.999722313 & 0.999499992 & 0.999277646 & 0.999055275 & 0.998832879 & 0.998610459 \\
\hline 0.09 & 1.000222507 & 1.000000223 & 0.999777914 & 0.999555580 & 0.999333223 & 0.999110840 & 0.998884333 & 0.998666000 \\
\hline
\end{tabular}

Table 5.5. Values of the Best Order Quantity $q^{*}(\mathrm{H}=5)$

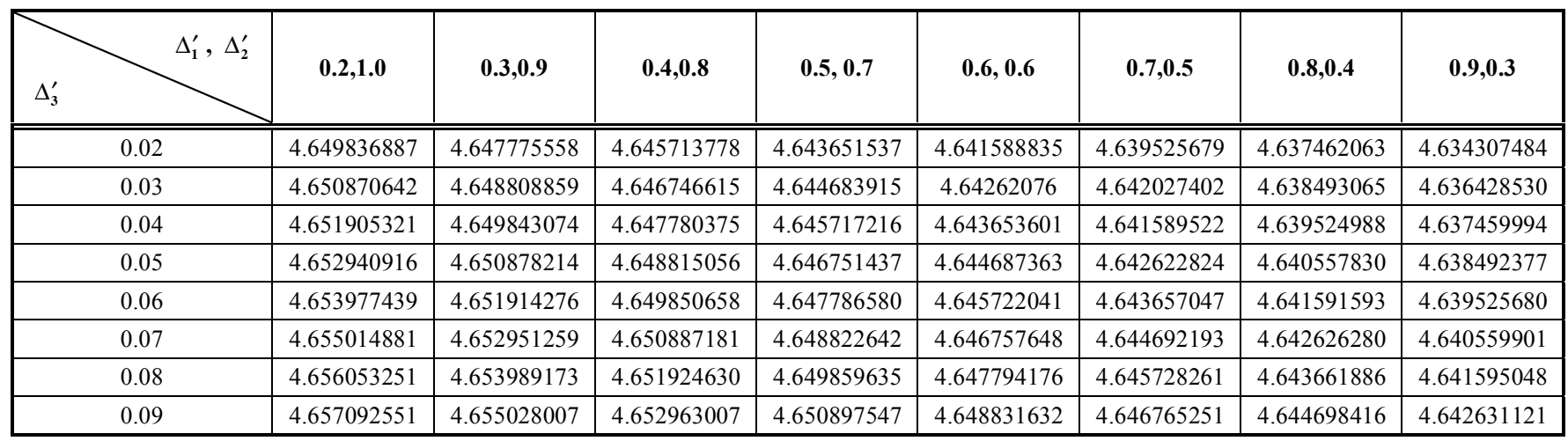

Table 5.5.a. Values of the Ratio $q^{\prime *}(\mathbf{H}=5)$

\begin{tabular}{|c|c|c|c|c|c|c|c|c|}
\hline & $0.2,1.0$ & $0.3,0.9$ & $0.4,0.8$ & $0.5,0.7$ & $0.6,0.6$ & $0.7,0.5$ & $0.8,0.4$ & $0.9,0.3$ \\
\hline 0.03 & 1.001999706 & 1.001555508 & 1.001111211 & 1.000666816 & 1.000222323 & 1.000094487 & 0.9993330230 & 0.9988882470 \\
\hline 0.04 & 1.002222621 & 1.001778323 & 1.001333928 & 1.000889434 & 1.000444841 & 1.000000149 & 0.9995553580 & 0.9991104690 \\
\hline 0.05 & 1.002445733 & 1.002001337 & 1.001556843 & 1.001112250 & 1.000667559 & 1.000222767 & 0.9997778770 & 0.9993328890 \\
\hline 0.07 & 1.002892555 & 1.002447961 & 1.002003269 & 1.001558478 & 1.001113588 & 1.000668599 & 1.0002235120 & 0.9997783240 \\
\hline 0.08 & 1.003116265 & 1.002671573 & 1.002226781 & 1.001781891 & 1.001336901 & 1.000891813 & 1.0004466260 & 1.0000013400 \\
\hline 0.09 & 1.003340175 & 1.002895383 & 1.002450492 & 1.002005502 & 1.001560414 & 1.001115226 & 1.0006699400 & 1.0002245550 \\
\hline
\end{tabular}


Table 5.6. Values of the Best Order Quantity $q$ * $(H=10)$

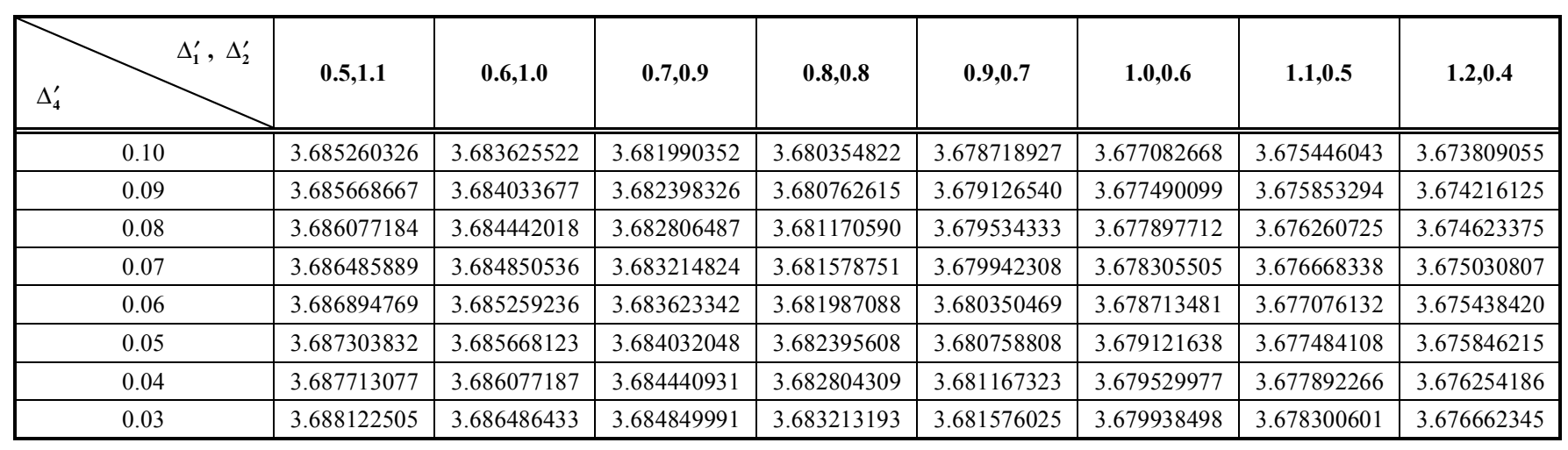

Table 5.6.a. Values of the Ratio $q^{1 *}(\mathbf{H}=10)$

\begin{tabular}{|c|c|c|c|c|c|c|c|c|}
\hline & $\Delta_{\mathbf{1}}^{\prime}, \Delta_{\mathbf{2}}^{\prime}$ & $\mathbf{0 . 5 , 1 . 1}$ & $\mathbf{0 . 6 , 1 . 0}$ & $\mathbf{0 . 7 , 0 . 9}$ & $\mathbf{0 . 8 , 0 . 8}$ & $\mathbf{0 . 9 , 0 . 7}$ & $\mathbf{1 . 0 , 0 . 6}$ & $\mathbf{1 . 1 , 0 . 5}$ \\
\hline & & & & & & $\mathbf{1 . 2 , 0 . 4}$ \\
\hline \hline 0.10 & 1.000222931 & 0.999779225 & 0.999335421 & 0.998891519 & 0.998447518 & 0.998003418 & 0.997559219 & 0.997114921 \\
\hline 0.09 & 1.000333759 & 0.999890003 & 0.999446150 & 0.999273611 & 0.998558963 & 0.998114000 & 0.997669751 & 0.997225405 \\
\hline 0.08 & 1.000444636 & 1.000000832 & 0.999556930 & 0.999112928 & 0.998668829 & 0.998224630 & 0.997780333 & 0.997335937 \\
\hline 0.07 & 1.000555630 & 1.000111709 & 0.999667757 & 0.999223708 & 0.998779558 & 0.998335310 & 0.997890964 & 0.997446519 \\
\hline 0.06 & 1.000666538 & 1.000222635 & 0.999778634 & 0.999334535 & 0.998890338 & 0.998446040 & 0.998001644 & 0.997557150 \\
\hline 0.05 & 1.000777562 & 1.000333612 & 0.999889561 & 0.999445412 & 0.999001166 & 0.998556818 & 0.998112373 & 0.997667830 \\
\hline 0.04 & 1.000888636 & 1.000444636 & 1.000000537 & 0.999556339 & 0.999112041 & 0.998667646 & 0.998223152 & 0.997778558 \\
\hline 0.03 & 1.000999760 & 1.000555711 & 1.000555711 & 1.000111561 & 0.999667314 & 0.998778524 & 0.998333979 & 0.997889337 \\
\hline
\end{tabular}

Table 5.7. Values of the Best Order Quantity $q^{*}(\mathrm{H}=15)$

\begin{tabular}{|c|c|c|c|c|c|c|c|c|}
\hline$\Delta_{3}^{\prime}, \Delta_{4}^{\prime}$ & 1.0 & 0.9 & 0.8 & 0.7 & 0.6 & 0.5 & 0.4 & 0.3 \\
\hline $0.04,0.3$ & 3.218544557 & 3.217831424 & 3.217118210 & 3.216404922 & 3.215691549 & 3.214978100 & 3.214264576 & 3.213550967 \\
\hline $0.05,0.2$ & 3.221156239 & 3.220442526 & 3.219728739 & 3.219014871 & 3.218300923 & 3.217586895 & 3.216872787 & 3.216158599 \\
\hline $0.06,0.1$ & 3.223776425 & 3.223062131 & 3.222347758 & 3.221633309 & 3.220918781 & 3.220204172 & 3.219489483 & 3.218774719 \\
\hline $0.07,0.09$ & 3.224253736 & 3.223539339 & 3.222824861 & 3.222110308 & 3.221395670 & 3.220680956 & 3.219966163 & 3.219251290 \\
\hline $0.08,0.08$ & 3.224731332 & 3.224016830 & 3.223302247 & 3.222587585 & 3.221872847 & 3.219012731 & 3.220443127 & 3.219728149 \\
\hline $0.09,0.07$ & 3.225209211 & 3.224494605 & 3.223779913 & 3.223065146 & 3.222350299 & 3.220920012 & 3.220920370 & 3.220205287 \\
\hline $0.1,0.06$ & 3.225687376 & 3.224972660 & 3.224257868 & 3.223542992 & 3.222828040 & 3.222113009 & 3.221397897 & 3.220682705 \\
\hline $0.2,0.05$ & 3.228322353 & 3.227503373 & 3.226891674 & 3.226176214 & 3.225460680 & 3.224745064 & 3.224029369 & 3.223313594 \\
\hline
\end{tabular}

Table 5.7.a. Values of the Ratio $q^{\prime *}(\mathrm{H}=15)$

\begin{tabular}{|c|c|c|c|c|c|c|c|c|}
\hline$\Delta_{3}^{\prime}, \Delta_{4}^{\prime}$ & 1.0 & 0.9 & 0.8 & 0.7 & 0.6 & 0.5 & 0.4 & 0.3 \\
\hline $0.04,0.3$ & 0.999928507 & 0.999706953 & 0.999485374 & 0.999263772 & 0.999042143 & 0.998820490 & 0.998598815 & 0.998377113 \\
\hline $0.05,0.2$ & 1.000739898 & 1.000518164 & 1.000296406 & 1.000074624 & 0.999852816 & 0.999630984 & 0.999409127 & 0.999187245 \\
\hline $0.06,0.1$ & 1.001553930 & 1.001332015 & 1.001110076 & 1.000888113 & 1.000666125 & 1.000444112 & 1.000222075 & 1.000000014 \\
\hline $0.07,0.09$ & 1.001702220 & 1.001480273 & 1.001258301 & 1.001036306 & 1.000814284 & 1.000592238 & 1.000370169 & 1.000148074 \\
\hline $0.08,0.08$ & 1.001850598 & 1.001628619 & 1.001406614 & 1.001184585 & 1.000962532 & 1.000073959 & 1.000518350 & 1.000296223 \\
\hline $0.09,0.07$ & 1.001999064 & 1.001777052 & 1.001555014 & 1.001332952 & 1.001110865 & 1.000666508 & 1.000666619 & 1.000444459 \\
\hline $0.1,0.06$ & 1.002147619 & 1.001925573 & 1.001703504 & 1.001481408 & 1.001259289 & 1.001037145 & 1.000814976 & 1.000592782 \\
\hline $0.2,0.05$ & 1.002966246 & 1.002711808 & 1.002521767 & 1.002299490 & 1.002077190 & 1.001854864 & 1.001632514 & 1.001410139 \\
\hline
\end{tabular}


Table 5.8. Values of the Best Order Quantity $q^{*}(\mathrm{H}=\mathbf{2 0})$

\begin{tabular}{|c|c|c|c|c|c|c|c|c|}
\hline$\Delta_{3}^{\prime}, \Delta_{4}^{\prime}$ & 0.9 & 0.8 & 0.7 & 0.6 & 0.5 & 0.4 & 0.3 & 0.2 \\
\hline $0.3,0.10$ & 2.922718577 & 2.923369555 & 2.924020459 & 2.924671287 & 2.925322046 & 2.925972730 & 2.926623344 & 2.927273888 \\
\hline $0.4,0.09$ & 2.924512860 & 2.925164233 & 2.925815536 & 2.926466764 & 2.927117923 & 2.927769011 & 2.928420025 & 2.929070963 \\
\hline $0.5,0.08$ & 2.926311550 & 2.926963328 & 2.927615032 & 2.928266665 & 2.928918224 & 2.929569707 & 2.930221121 & 2.93087246 \\
\hline $0.6,0.07$ & 2.928114680 & 2.928766859 & 2.929418964 & 2.930070993 & 2.930722953 & 2.931374842 & 2.932026657 & 2.932678402 \\
\hline $0.7,0.06$ & 2.929922259 & 2.930574840 & 2.931227346 & 2.931879782 & 2.932532144 & 2.933184435 & 2.933836651 & 2.934488798 \\
\hline $0.8,0.05$ & 2.931734311 & 2.932387294 & 2.933040208 & 2.933693046 & 2.934345810 & 2.934998504 & 2.935651123 & 2.936303672 \\
\hline $0.9,0.04$ & 2.933550854 & 2.934204241 & 2.934857558 & 2.935510800 & 2.936163972 & 2.936817069 & 2.937470096 & 2.938123048 \\
\hline $1.0,0.03$ & 2.935371909 & 2.936025705 & 2.936679426 & 2.937330710 & 2.937986647 & 2.938640154 & 2.939293580 & 2.939946941 \\
\hline
\end{tabular}

Table 5.8.a. Values of the Ratio $q^{\prime *}(\mathbf{H = 2 0})$

\begin{tabular}{|c|c|c|c|c|c|c|c|c|}
\hline$\Delta_{3}^{\prime}, \Delta_{4}^{\prime}$ & 0.9 & 0.8 & 0.7 & 0.6 & 0.5 & 0.4 & 0.3 & 0.2 \\
\hline $0.3,0.10$ & 0.999389146 & 0.999611741 & 0.999834310 & 1.000056853 & 1.002793720 & 1.000501866 & 1.000724336 & 1.000946782 \\
\hline $0.4,0.09$ & 1.000002681 & 1.000225410 & 1.000448115 & 1.000670795 & 1.000893451 & 1.001116083 & 1.001338690 & 1.00156127 \\
\hline $0.5,0.08$ & 1.000617722 & 1.000830315 & 1.001063421 & 1.00128625 & 1.001509043 & 1.001731810 & 1.001954553 & 1.002177271 \\
\hline $0.6,0.07$ & 1.003715805 & 1.001457286 & 1.001680265 & 1.001903219 & 1.002126149 & 1.002349055 & 1.002571935 & 1.002794792 \\
\hline $0.7,0.06$ & 1.001852361 & 1.002075503 & 1.002298620 & 1.002521713 & 1.002744780 & 1.002967824 & 1.003190841 & 1.003413835 \\
\hline $0.8,0.05$ & 1.002471971 & 1.002695051 & 1.002918507 & 1.003141737 & 1.003364942 & 1.003588123 & 1.003811278 & 1.004034410 \\
\hline $0.9,0.04$ & 1.003093116 & 1.003316534 & 1.003539928 & 1.003763297 & 1.003986641 & 1.004209960 & 1.004433255 & 1.004656524 \\
\hline $1.0,0.03$ & 1.003715805 & 1.003939362 & 1.004162886 & 1.004385593 & 1.004609883 & 1.004833342 & 1.005056774 & 1.005280183 \\
\hline
\end{tabular}

Table 5.9. Values of the Best Order Quantity $q^{*}(\mathrm{H}=\mathbf{2 5})$

\begin{tabular}{|c|c|c|c|c|c|c|c|c|}
\hline$\Delta_{3}^{\prime}, \Delta_{4}^{\prime}$ & $0.2,0.25$ & $0.3,0.3$ & $0.4,0.35$ & $0.5,0.4$ & $0.6,0.45$ & $0.7,0.5$ & $0.8,0.55$ & $0.9,0.6$ \\
\hline $0.02,0.025$ & 2.714658890 & 2.714357303 & 2.714055697 & 2.713754075 & 2.713452439 & 2.713150788 & 2.712849116 & 2.712547430 \\
\hline $0.03,0.030$ & 2.714719211 & 2.714417620 & 2.714116008 & 2.713814382 & 2.713512736 & 2.713211079 & 2.712909398 & 2.712607706 \\
\hline $0.04,0.035$ & 2.714779541 & 2.714477940 & 2.714176323 & 2.713874692 & 2.713573040 & 2.713271374 & 2.712969687 & 2.712667991 \\
\hline $0.05,0.040$ & 2.714839879 & 2.714538272 & 2.714236646 & 2.713935004 & 2.713633348 & 2.713331676 & 2.713029985 & 2.712728278 \\
\hline $0.06,0.045$ & 2.714900219 & 2.714598603 & 2.714296971 & 2.713995325 & 2.711860384 & 2.713512589 & 2.713090285 & 2.712788569 \\
\hline $0.07,0.05$ & 2.714960563 & 2.714658942 & 2.714357305 & 2.714055653 & 2.716109593 & 2.713452295 & 2.713150589 & 2.712848867 \\
\hline $0.08,0.055$ & 2.715020915 & 2.714719288 & 2.714417642 & 2.714115985 & 2.713814304 & 2.713512612 & 2.713210900 & 2.712909174 \\
\hline $0.09,0.06$ & 2.715081270 & 2.714779638 & 2.714477987 & 2.714176320 & 2.713874633 & 2.713572937 & 2.713271215 & 2.712969484 \\
\hline
\end{tabular}

Table 5.9.a. Values of the Ratio $q^{\prime *}(\mathbf{H}=\mathbf{2 5})$

\begin{tabular}{|c|c|c|c|c|c|c|c|c|}
\hline$\Delta_{3}^{\prime}, \Delta_{4}^{\prime}$ & $0.2,0.25$ & $0.3,0.3$ & $0.4,0.35$ & $0.5,0.4$ & $0.6,0.45$ & $0.7,0.5$ & $0.8,0.55$ & $0.9,0.6$ \\
\hline $0.02,0.025$ & 0.999911142 & 0.999800056 & 0.999688963 & 0.999577864 & 0.999466760 & 0.999355651 & 0.999244534 & 0.999133412 \\
\hline $0.03,0.030$ & 0.999933360 & 0.999822273 & 0.999711178 & 0.999600078 & 0.999488970 & 0.999400067 & 0.999266738 & 0.999155613 \\
\hline $0.04,0.035$ & 0.999955582 & 0.999844491 & 0.999733394 & 0.999622292 & 0.999511182 & 0.999400067 & 0.999288945 & 0.999177819 \\
\hline $0.05,0.040$ & 0.999977807 & 0.999866714 & 0.999755613 & 0.999644507 & 0.999533396 & 0.999422279 & 0.999311155 & 0.999200025 \\
\hline $0.06,0.045$ & 1.000000033 & 0.999888936 & 0.999777833 & 0.999666726 & 0.998880347 & 0.999488916 & 0.999333365 & 0.999222232 \\
\hline $0.07,0.05$ & 1.000022260 & 0.999911161 & 0.999800057 & 0.999688947 & 1.000445491 & 0.999466707 & 0.999355578 & 0.999244442 \\
\hline $0.08,0.055$ & 1.000044401 & 0.999933389 & 0.999822281 & 0.999711169 & 0.999600049 & 0.999488924 & 0.999377793 & 0.999266655 \\
\hline $0.09,0.06$ & 1.000066721 & 0.999955618 & 0.999844508 & 0.999733393 & 0.999622270 & 0.999511144 & 0.999400009 & 0.99928887 \\
\hline
\end{tabular}


Table 6.1. Simulated Sampling Distribution

\begin{tabular}{|c|c|c|c|c|c|}
\hline $\mathbf{H}$ & $\begin{array}{c}\text { Sample Size } \\
n\end{array}$ & $\underset{\bar{x}}{\operatorname{Mean}}$ & $\begin{array}{c}\text { Standard Deviation } \\
\sigma\end{array}$ & Skewness & Kurtosis \\
\hline \multirow{9}{*}{5} & 10 & 4.365199 & 0.162544 & 0.016865 & 1.851675 \\
\hline & 25 & 4.427767 & 0.134044 & 0.290297 & 2.139292 \\
\hline & 50 & 4.372178 & 0.181885 & 0.177548 & 2.252878 \\
\hline & 75 & 4.389462 & 0.220237 & 0.378736 & 4.141036 \\
\hline & 100 & 4.356456 & 0.157625 & 0.018133 & 2.633181 \\
\hline & 150 & 4.352150 & 0.169417 & 0.068435 & 2.043601 \\
\hline & 200 & 4.370565 & 0.169089 & 0.111821 & 2.576298 \\
\hline & 250 & 4.365365 & 0.172009 & 0.037441 & 2.470689 \\
\hline & 300 & 4.358217 & 0.168921 & 0.045316 & 2.293105 \\
\hline \multirow{9}{*}{10} & 10 & 3.577240 & 0.073720 & 0.030391 & 1.901382 \\
\hline & 25 & 3.610458 & 0.061027 & 0.486862 & 2.446707 \\
\hline & 50 & 3.582542 & 0.088321 & 0.197897 & 2.345901 \\
\hline & 75 & 3.587596 & 0.097364 & 0.090387 & 3.284802 \\
\hline & 100 & 3.574557 & 0.073196 & 0.099806 & 2.771479 \\
\hline & 150 & 3.571897 & 0.079265 & 0.130015 & 2.182581 \\
\hline & 200 & 3.580865 & 0.078057 & 0.244178 & 2.665714 \\
\hline & 250 & 3.578076 & 0.079543 & 0.099518 & 2.484007 \\
\hline & 300 & 3.575679 & 0.079128 & 0.093955 & 2.290149 \\
\hline \multirow{9}{*}{15} & 10 & 3.161705 & 0.045506 & 0.059349 & 1.972329 \\
\hline & 25 & 3.184784 & 0.038528 & 0.576960 & 2.689910 \\
\hline & 50 & 3.166326 & 0.057486 & 0.155727 & 2.352791 \\
\hline & 75 & 3.168448 & 0.059877 & 0.051294 & 3.132471 \\
\hline & 100 & 3.160770 & 0.046183 & 0.136328 & 2.904075 \\
\hline & 150 & 3.158833 & 0.050046 & 0.148548 & 2.270863 \\
\hline & 200 & 3.164713 & 0.048818 & 0.294278 & 2.782167 \\
\hline & 250 & 3.162767 & 0.049854 & 0.108460 & 2.551390 \\
\hline & 300 & 3.161759 & 0.050093 & 0.088725 & 2.318178 \\
\hline \multirow{9}{*}{20} & 10 & 2.890062 & 0.032384 & 0.088654 & 2.057577 \\
\hline & 25 & 2.908086 & 0.028322 & 0.577664 & 2.830716 \\
\hline & 50 & 2.894277 & 0.042709 & 0.105012 & 2.331451 \\
\hline & 75 & 2.895203 & 0.042548 & 0.044281 & 3.097650 \\
\hline & 100 & 2.889886 & 0.033550 & 0.131523 & 3.002758 \\
\hline & 150 & 2.888345 & 0.036281 & 0.142627 & 2.334399 \\
\hline & 200 & 2.892733 & 0.035116 & 0.297848 & 2.874302 \\
\hline & 250 & 2.891209 & 0.035925 & 0.091979 & 2.616260 \\
\hline & 300 & 2.890813 & 0.036414 & 0.063366 & 2.346792 \\
\hline \multirow{9}{*}{25} & 10 & 2.692876 & 0.025068 & 0.109245 & 2.137106 \\
\hline & 25 & 2.707886 & 0.022749 & 0.520967 & 2.862945 \\
\hline & 50 & 2.696806 & 0.034220 & 0.062101 & 2.300172 \\
\hline & 75 & 2.697121 & 0.032866 & 0.047985 & 3.097250 \\
\hline & 100 & 2.693099 & 0.026466 & 0.101543 & 3.070260 \\
\hline & 150 & 2.691804 & 0.028515 & 0.122223 & 2.379015 \\
\hline & 200 & 2.695325 & 0.027425 & 0.271612 & 2.936914 \\
\hline & 250 & 2.694053 & 0.028093 & 0.065316 & 2.670536 \\
\hline & 300 & 2.693983 & 0.028692 & 0.035267 & 2.373990 \\
\hline
\end{tabular}


or $\Delta_{1}^{\prime}$, the best order size is an increasing function of $\Delta_{3}^{\prime}$ and $\Delta_{4}^{\prime}$, while it is a decreasing or an increasing function of $\Delta_{2}^{\prime}$ or $\Delta_{1}^{\prime}$ respectively. Table 5.9 shows that for a fixed value of $\Delta_{1}^{\prime}$ and $\Delta_{2}^{\prime}$, the best order size is an increasing function of $\Delta_{3}^{\prime}$ and $\Delta_{4}^{\prime}$, while it is a decreasing function of $\Delta_{1}^{\prime}$ and $\Delta_{2}^{\prime}$ for fixed $\Delta_{3}^{\prime}$ and $\Delta_{4}^{\prime}$.

Entries in Tables 5.1(a) to 5.9(a) supplemented by calculations of similar values of the ratio $q^{\prime *}$ point to possible robustness of the optimal order quantity when the decision parameters $\Delta_{1}, \Delta_{2}, \Delta_{3}$ and $\Delta_{4}$ are under, over or equally estimated.

\section{CONCLUSION}

An inventory model under flexibility and reliability criteria is considered in a non-random optimization environment. For highly demanded quality products normally, planning horizon is taken as finite and of crisp in nature. But in reality due to rapid change of environment, demand for the items fluctuates every year. So it is worthwhile to consider the planning horizon as uncertain in nature. Here stochastic nature of the uncertain planning horizon is considered. Complexities of the models increase when some other parameters of the model become imprecise in non-stochastic sense that is fuzzy in nature. Due to constraints of the system, the average level of investment necessary to operate effectively is assumed to be fixed in managing the long term investment in inventory management. Clamping by the backdrop of many factors, the system cost seems to be considered fixed. The methodology presented here shows that the total variable cost in the same industry with the crisp and fuzzy are almost the same. The optimal solution will be a good approximation with fuzzy strategy than that of crisp, when flexibility and reliability criteria are considered.

For giving managerial insights into the non-random optimization approach, a sensitivity analysis is performed to determine the sceneries which would result in vibration of the setup cost and inventory holding cost. It is examined that the total cost corresponding to our fuzzy solution in presence of reliability criteria is $0.0256 \%$ more consistent than the cost obtained by the crisp. The proposed approach continues to work dynamically as the market evolves; it makes sense from our experience to conclude that this model can be an effective tool for dynamic inventory models.

Simulated sampling distribution converges around skewness of zero and kurtosis of three. In Table $\mathbf{6 . 1}$ the results obtained from the data admitting normality are what would be expected. The conclusion drawn from this analysis is that managers making financial aid decisions are better equipped to make informed decisions using vibrated values of decision parameters during implementations of the model.

In order to provide an assessment of the effectiveness of the present paper, further research should be included to a comprehensive numerical investigation of real life situations and identify the nature of system cost and empirically test more extricable for these effect of the values of the firm. With vibration in the system cost, the risk of the total cash flow can be reduced and hence the risk implication of the altering system cost may now be ignored.

There have been many methods to be proposed for the modelling in a non-random optimization environment with a disposal mechanism under flexibility and reliability criteria. However they cannot meet the real choice process with performance evaluation because most of the methods are the mathematical models. This is real critical subject that can result in choosing a disposal mechanism with various performances. The most important part of this, it gives a concrete result by recording the expert's previous experiences and process this with fuzzy logic arithmetic. As a future study it is possible to develop this method by using numerical performances criteria (DEA) to use objective and subjective evaluations together.

Also further research should include a comprehensive investigation of real life system and identify the nature of the system cost and empirically test most extricable for their effect. Further this model can be extended to consider the time value of money. Future study will incorporate more realistic propositions.

\section{APPENDIX-A}

$$
\begin{aligned}
& A=\frac{q^{*}}{r^{* 3}}\left(H+\phi_{2}\right)+a \lambda^{*-(b+1)}\left\{-2 c\left(1-r^{*}\right)^{c-1}+r^{*} c(c-1)\left(1-r^{*}\right)^{c-2}\right\} \\
& B=\frac{2 r^{*}}{q^{*} \lambda^{* 3}}\left(S+\phi_{1}\right)+a(b+1)(b+2) r^{*}\left(1-r^{*}\right)^{c} \lambda^{*-(b+3)} \\
& C=2 \lambda^{*} q^{*}\left(H+\phi_{2}\right) \\
& D=-\frac{1}{\lambda^{* 2} q^{*}}\left(S+\phi_{1}\right)-a\left[\left(1-r^{*}\right)^{c}-r^{*} c\left(1-r^{*}\right)^{(c-1)}\right] \times(b+1) \lambda^{*-(b+2)} \\
& E=-2 \lambda^{*} q^{*}\left(H+\phi_{2}\right)+2 r^{* 2} \lambda^{*-b} a\left[\left(1-r^{*}\right)^{c}-r^{*} c\left(1-r^{*}\right)^{c-1}\right] \\
& F=\frac{r}{\lambda^{* 2} q^{* 2}}\left(S+\phi_{1}\right) \\
& G=\left(S+\phi_{1}\right)+a(b+1) \lambda^{*-b} q^{*} \times\left[\left(1-r^{*}\right)^{c}-r^{*} c\left(1-r^{*}\right)^{(c-1)}\right] \\
& I=\frac{-1}{\lambda^{*} q^{* 2}}\left(S+\phi_{1}\right)-\frac{1}{2 r^{* 2}}\left(H+\phi_{2}\right) \\
& J=q^{* 2}\left(H+\phi_{2}\right) \\
& \text { Relative Error }(\mathrm{R} . \mathrm{E} .)=\left(\left(M^{*}-Z_{1}\right) / Z_{1}\right) \times 100 \\
& q^{*}=\frac{q^{\prime}}{q^{*}}
\end{aligned}
$$

\section{ACKNOWLEDGEMENT}

We would like to acknowledge Prof. D. Acharya, Director, IIT Kharagpur and Prof. K.S. Chaudhury, Department of Mathematics, Jadavpur University and Prof. M. Basu, Secretary, O.R.S.I., India whose works have 
incited us in our research area. Also we thank the anonymous referees for their contribution to improve the paper.

\section{REFERENCES}

[1] Silver EA. Operation research in inventory management: a review and critique. Oper Res 1981; 29: 628-45.

[2] Silver EA, Peterson R. Decision systems for inventory management and production planning. New York: John Wiley \& Sons 1985.

[3] Urgelleitti TG. Inventory control models and problems. Eur J Oper Res 1983; 14: 1-12.

[4] Wagner HM. Research portfolio for inventory management and production planning systems. Oper Res 1980; 28: 225-75.

[5] Whitin TM. Inventory control research: a survey. Manag Sci 1954; 1: 32-40.

[6] Cheng TCE. An economic production quantity model with flexibility and reliability considerations. Eur J Oper Res 1989; 39: 174-9.

[7] Cheng TCE. EPQ with process capability and quality assurance considerations. J Oper Res Soc 1991; 42: 713-20.
[8] Hadley G, Whitin TM. Analysis of inventory systems. Newzealand: Prentice-Hall Englewood Cliffs 1963.

[9] Porteus EL. Optimal lot sizing process quality improvement and setup cost reduction. Oper Res 1986; 34: 137-44.

[10] Rosenblatt MJ, Lee HL. Economic production cycles with imperfect production processes. Int Ind Eng Trans 1986; 14: 48-55.

[11] Tapiero CS, Ritchken PH, Reisman A. Reliability, pricing and quality control. Eur J Oper Res 1987; 31: 37-45.

[12] Tripathy PK, Wee WM, Majhi PR. An EOQ model with process reliability considerations. J Oper Res Soc 2003; 54: 549-54.

[13] Lee HM, Yao JS. Economic production quantity for fuzzy demand quantity and fuzzy production quantity. Eur J Oper Res 1998; 109: 203-11.

[14] Vujosevic M, Petrovic D, Petrovic R. EOQ formula when inventory cost is fuzzy. Int J Prod Econ 1996; 45: 499-504.

[15] Zimmermann HJ. Fuzzy set theory and its application. Second revised edition. Dordrecht: Kulwer Academic Publishers 1991.

[16] Tripathy PK, Pattnaik M. Optimization in an inventory model with reliability consideration. Appl Math Sci 2009; 3: 11-25.

[17] Mahata GC, Goswami A. Production lot size model with fuzzy production rate and fuzzy demand rate for deteriorating item under permissible delay in payments. J Oper Res Soc Ind 2006; 43: 35975 .

This is an open access article licensed under the terms of the Creative Commons Attribution Non-Commercial License (http://creativecommons.org/licenses/by-nc/ $3.0 /$ ) which permits unrestricted, non-commercial use, distribution and reproduction in any medium, provided the work is properly cited. 